TRANSACTIONS OF THE

AMERICAN MATHEMATICAL SOCIETY

Volume 351, Number 6, Pages 2295-2323

S 0002-9947(99)02151-0

Article electronically published on February 5, 1999

\title{
FAREY POLYTOPES AND CONTINUED FRACTIONS ASSOCIATED WITH DISCRETE HYPERBOLIC GROUPS
}

\author{
L. YA. VULAKH
}

\begin{abstract}
The known definitions of Farey polytopes and continued fractions are generalized and applied to diophantine approximation in $n$-dimensional euclidean spaces. A generalized Remak-Rogers isolation theorem is proved and applied to show that certain Hurwitz constants for discrete groups acting in a hyperbolic space are isolated. The approximation constant for the imaginary quadratic field of discriminant -15 is found.
\end{abstract}

\section{INTRODUCTION}

Let $V$ be the $n$-dimensional Euclidean space $\mathbf{R}^{n}$. The upper half-space $H^{n+1}=$ $\{(z, t): z \in V, t>0\}$ with the metric $d s^{2}=t^{-2}\left(|d z|^{2}+d t^{2}\right)$ can be used as a model of the $(n+1)$-dimensional hyperbolic space. Here $|\cdot|$ is Euclidean length in $V$. Let $\operatorname{Con}(n)$ denote the group of orientation-preserving isometries of $H^{n+1}$. Let $G$ be a geometrically finite discrete subgroup of $\operatorname{Con}(n)$ (see [1]). A geodesic in $H^{n+1}$ is a semicircle or a ray which is orthogonal to $V$. An element $g \in \operatorname{Con}(n)$ extends to a conformal transformation of $V \cup H^{n+1}$, the closure of $H^{n+1}$. Hence, $g$ will fix a point either in $H^{n+1}$ or on its boundary $V$. The type of $g$ is elliptic, parabolic or loxodromic depending on whether it has a fixed point in $H^{n+1}$, a single fixed point in $V$, or exactly two fixed points in $V$ (see e.g. [1]). If $g$ is loxodromic, the geodesic connecting its fixed points is called the axis of $g$. The transformation $g$ is hyperbolic if it is loxodromic and every plane containing its axis is $g$-invariant. We denote by $\mathcal{P}$ the set of parabolic fixed points (cusps) of $G$. In the sequel, we assume that $\infty \in \mathcal{P}$.

Let $P$ be a Dirichlet polygon of $G_{\infty}=\operatorname{Stab}(\infty, G)$ in $V$. Denote $P_{\infty}=\{(z, t) \in$ $\left.H^{n+1}: z \in P\right\}$. The region

$$
D=P_{\infty} \cap\left\{x \in H^{n+1}:\left|g^{\prime}(x)\right|<1, g \in G\right\}
$$

is an isometric fundamental domain for $G$ in $H^{n+1}$ (see [1] or [2]). Here $g^{\prime}(x)$ stands for the Jacobian of the transformation $g$. Denote

$$
K=K(\infty)=G_{\infty} \bar{D}, \quad K(u)=g K(\infty),
$$

where $u=g(\infty)$. Let $\partial K$ be the boundary of $K$. We shall say that $\partial K \cap \bar{D}$ is the floor of $D$. In the sequel, we shall be mainly concerned with the components of $\partial K$ (and $D$ ) of dimensions 0,1 , and $n$. We shall call them vertices (or cusps), edges, and faces of $K$ respectively. The vertices (and edges) of $K$ which belong

Received by the editors February 26, 1996 and, in revised form, May 19, 1997.

1991 Mathematics Subject Classification. Primary 11J99.

Key words and phrases. Diophantine approximation, Clifford algebra, hyperbolic geometry.

(C)1999 American Mathematical Society 
to $\bar{D}$ will be called the vertices (and edges) of $D$. For any region $R$ in $H^{n+1}$, the components of the boundary of $R$ of dimension $n$ which lie in vertical planes will be called the vertical faces of $R$. (Note that, in general, according to these definitions, the components of the boundary of $D$ of dimension 0 (or 1 ) which lie in the vertical faces of $D$ are not vertices (or edges) of $D$.)

Let $\alpha$ be a real irrational number. In $1891 \mathrm{~A}$. Hurwitz [17] showed that the inequality

$$
\left|\alpha-a c^{-1}\right|<\frac{1}{k|c|^{2}}
$$

has infinitely many solutions in coprime integers $a$ and $c$ when $k=\sqrt{5}$, and $\sqrt{5}$ is the best constant possible. The first geometric proof of this result was obtained by L. Ford in [12] where he makes use of properties of the modular group. Let $O_{d}$ be the ring of integers of the imaginary quadratic number field $\mathbf{Q}(\sqrt{-d})$. In 1925 Ford [13] applied his approach to the Picard group to show that for any $\alpha \notin \mathbf{Q}(\sqrt{-1})$ (3) has infinitely many solutions in coprime $a, c \in O_{1}$ when $k=\sqrt{3}$, and $\sqrt{3}$ is the best constant possible. A modification of the Ford geometric approach is developed in [35] where the approximation constants for the fields $\mathbf{Q}(\sqrt{-5})$ and $\mathbf{Q}(\sqrt{-6})$ are found.

Let $\alpha \in V-\mathcal{P}$. Assume that $\infty \in \bar{D}$, where $\bar{D}$ is the closure of $D$. It is known (see e.g. [1] or [3]) that there is a constant $k>0$, depending only on $G$, such that the inequality

$$
|\alpha-g(\infty)|<r^{2}(g) / k
$$

holds for infinitely many left cosets of $G_{\infty}$ in $G$. Here $r(g)$ is the radius of the isometric sphere $I(g)=\left\{x \in H^{n+1}:\left|g^{\prime}(x)\right|=1\right\}$ of $g \in G$. If $n=1$ and $G$ is the modular group, (4) is reduced to (3).

For a fixed $\alpha \in V-\mathcal{P}$, we denote by $k(\alpha)$ the supremum of all such $k$ in (4). The set of numbers

$$
\mathcal{L}(G)=\{1 / k(\alpha), \alpha \in V-\mathcal{P}\}
$$

is called the Lagrange spectrum for $G$ and $C(G)=\sup \mathcal{L}(G)$ the Hurwitz constant for $G$.

For an oriented geodesic $L$ in $H^{n+1}$ with the initial and terminal endpoints $\eta^{\prime}, \eta \in V$ respectively we write $L=\left(\eta^{\prime}, \eta\right)$. In particular, $(\infty, \eta)$ is a vertical ray in $H^{n+1}$ through $\eta$. We shall say that $h t(L)=\left|\eta^{\prime}-\eta\right| / 2=h_{L} / 2$ is the height of $L$ and denote

$$
k(L)=\sup h_{g L}=\sup \left|g(\eta)-g\left(\eta^{\prime}\right)\right|, g \in G .
$$

If $L$ is the axis of a loxodromic element in $G$, then $k(L)=k\left(\eta^{\prime}\right)=k(\eta)$. Otherwise, this is not always true (see e.g. [11]). The set of numbers

$$
\mathcal{M}(G)=\left\{1 / k(L), L \subset H^{n+1}\right\}
$$

is called the Markov spectrum for $G$.

Let $D$ be an isometric fundamental domain of $G$. Let an edge $\sigma$ of $D$ lie on a geodesic $L$ which is not a vertical ray. The point of $L$ farthest from $V$ is called the summit of $\sigma$.

Define $k_{G}$ to be the largest value of $k$ such that the connected parts of $D$ lying below $t=k / 2$ are pyramidal regions bounded by the faces of $D$ which meet at a vertex or cusp of $D$ and the Euclidean plane $t=k / 2$. If the summit of every edge of $D$ belongs to the closure of $D$, then $k_{G}$ is twice the distance from $V$ to the set 
of summits of $D$. The following theorem is proved in [36] (see also Theorems 9 and $17)$.

Theorem 1. Let $G$ be a geometrically finite group acting in the $(n+1)$-dimensional hyperbolic space $H^{n+1}$. Let the $n$-dimensional Euclidean space $V$ be the limit set and $\mathcal{P}$ the set of parabolic fixed points of $G$. Suppose that $\infty \in \mathcal{P}$. Let $\alpha \in V-\mathcal{P}$. Then there are infinitely many left cosets of $G_{\infty}$ in $G$ whose members $g$ satisfy (4) with $k=k_{G}$. Thus, $C(G) \leq 1 / k_{G}$.

In $\S 2$, we introduce the fundamental notion of a $v$-cell $N(v)$ for every vertex or cusp of a fundamental domain for $G$. The $k$-neighborhood of $v$ introduced in [34], [35], and [36] is a subset of $N(v)$, so that $N(v)$ is the $\infty$-neighborhood of the vertex. The Farey tessellation is a tessellation of $H^{n+1}$ by $v$-cells. It is $G$-invariant. For the modular group, this definition was introduced by C. Series in [26]. A Farey polytope in $V$ is the projection of a non-vertical face of a $v$-cell from $\infty$ into $V$. For some Bianchi groups, Farey polygons with three, four and six sides were introduced by A. L. Schmidt in [21] and [24] (see also [22]) using another definition. Here, the basic properties of the Farey polytopes are established (Theorems 5 and 9).

In $\S 3$, Algorithm I generating the best possible approximations of $\alpha \in V$ by the cusps $g \propto, g \in G$, is introduced. It is analogous to but different from the standard continued fraction algorithm and the algorithms introduced by A. L. Schmidt in [23], [24], and [25]. Here, some of the known applications of the continued fraction algorithms are discussed. In particular, Algorithm I is applied to develop the reduction theory for geodesics in $H^{n+1}$ which is similar to the reduction theory of binary indefinite quadratic forms.

In $\S 4$, the notion of an extremal geodesic is introduced. (A geodesic $L=\left(\eta^{\prime}, \eta\right.$ ) is extremal if $k(L)=\left|\eta^{\prime}-\eta\right|$.) If an extremal geodesic exists in the $G$-orbit of a geodesic, Algorithm I can be used to find it since the set of reduced geodesics contains an extremal one in that case. Simple criteria of extremality of a geodesic are given in Corollary 24 and Lemma 25. In Examples 27, 28, and 31, they are applied to Bianchi groups $P G L_{2}\left(O_{d}\right), d \equiv 3(\bmod 4), d \leq 19$, and to the extended Bianchi group $B_{15}$. In all the known cases (see e.g. [35]), among the extremal geodesics found there is a geodesic $L$ such that the Hurwitz constant $C(G)=$ $1 / k(L)$. For the group $B_{15}$, we find that $1 / \sqrt{2} \leq C\left(B_{15}\right)<2 / \sqrt{7}$. (It is proved in $\S 5$ that $C\left(B_{15}\right)=1 / \sqrt{2}$ so that in the only unknown case of $d=15$ such an extremal geodesic has been found too.) Applying Algorithm I to the extremal geodesics found, we also find the set of reduced geodesics. In conclusion, we prove that if the endpoints $v$ and $v^{\prime}$ of a critical edge $\sigma$ of $D$ are cusps and there is a reflection $R \in G$ such that $v^{\prime}=R(v)$, then the Hurwitz constant $C(G)=k(\sigma)=\left|v^{\prime}-v\right|$ and it is an accumulation point in the Lagrange and Markov spectra (Corollary 30). These results are applied to show that the inequality (3) has infinitely many solutions in coprime $a, c \in O_{15}$ when $k=1 / 2$, and $1 / 2$ is the best constant possible. Thus, the Hurwitz constant $C(G)=2, G=P G L_{2}\left(O_{15}\right)$, and it is not isolated in $\mathcal{L}(G)$ (Example 31). In Example 32, a similar result is obtained for the set of integral quaternions whose approximation constant is 1 (see [36]).

It is shown in [35] that the strict inequality $C(G)<1 / k_{G}$ holds in Theorem 1 for a Bianchi group $G=B_{d}$ when $d \equiv 3(\bmod 4), d \leq 19, d \neq 15, \operatorname{though} C(G)=1 / k_{G}$ when $d=1,2,5,6$. In Example 28, it is found that $1 / \sqrt{2} \leq C(G)<1 / k_{G}=2 / \sqrt{7}$ when $d=15$. In $\S 5$, applying the results obtained in $\S 2$ and $\S 4$ we find that 
$C\left(B_{15}\right)=1 / \sqrt{2}$ which is the approximation constant for the field $\mathbf{Q}(\sqrt{-15})$. The result obtained can be represented as follows.

Theorem 2. Let $\alpha \in \mathbf{C}-\mathbf{Q}(\sqrt{-15})$. The inequality

$$
\left|\alpha-\frac{a}{c}\right|<\frac{n(a, c)}{k|c|^{2}}
$$

has infinitely many solutions in $a, c \in \mathcal{O}_{15}$ when $k=\sqrt{2}$, whereas for $\alpha=$ $(\omega+\sqrt{\omega}) / 2$ and $(\omega+\sqrt{-\omega}) / 2$ the inequality holds only for a finite number of $a / c \in \mathbf{Q}(\sqrt{-15})$ if $k>\sqrt{2}$. Here $\mathcal{O}_{15}$ stands for the ring of integers in $\mathbf{Q}(\sqrt{-15})$ and $n(a, c)$ for the norm of the ideal generated by $a$ and $c$.

In $\S 6$, we prove an isolation theorem (Theorem 36) which generalizes to $n$ dimensional euclidean spaces the isolation theorems proved in [8], p. 25, and [31] for $n=1$ and 2. In contrast to the case mentioned above when the endpoints of $\sigma$ are cusps of $D$ and the Hurwitz constant $C(G)$ is a limit point in the Lagrange and Markov spectra of $G$, the isolation theorem implies, in all the known cases, that $C(G)$ is isolated provided the critical edges $\sigma$ of $D$ lie on the extremal geodesics which are the axes of loxodromic elements in $G$. Here it is shown for the first time that the approximation constants for the imaginary quadratic fields $\mathbf{Q}(\sqrt{-5})$ and $\mathbf{Q}(\sqrt{-6})$, which are found in [35], and for the three-dimensional euclidean space (see [36], Example 3) are isolated.

\section{Farey tessellation and Farey polytopes}

In this section, we first introduce the notion of a $v$-component and a $v$-cell for every vertex and cusp $v$ of $D$. A $k$ - neighborhood of $v$ (see [34], [35], [36]) is a subset of the $v$-cell, so that the $v$-cell is the $\infty$-neighborhood of $v$. Then the definition of a Farey polytope in $V$ as the projection of a non-vertical face of a $v$-cell from $\infty$ into $V$ is given. It is shown (see Examples 7 and 11) that the Farey polygons introduced by A. L. Schmidt in [21] and [24] are covered by this definition. We also introduce the definition of the Farey tessellation of $H^{n+1}$ by $v$-cells. For the modular group it was first introduced by C. Series in [26].

Let $\phi_{i}$ be a face of $K$ (see (2)) which lies on the isometric sphere of $g_{i} \in G$ with center $u_{i}=g_{i}(\infty)$. The projection of $\phi_{i}$ from $\infty$ into $V$ is a polytope $p\left(u_{i}\right)$. The polytopes $p\left(u_{i}\right)$ form a tessellation of $V$. Let $v_{V}$ be the projection of a vertex $v$ of $K$. Suppose that it is the common vertex of the polytopes $p\left(u_{i}\right), i=1, \ldots, r$. Denote by $A\left(v_{V}\right)$ the convex hull of the points $u_{i}, i=1, \ldots, r$, in $V$. (Note that if all the faces of $K$ are congruent modulo $G_{\infty}$, then the polytope $p\left(u_{i}\right)$ is the Voronoi cell of $u_{i}$ and $A\left(v_{V}\right)$, as defined above, is the Delaunay cell of $v_{V}$ (see [9], 33-35).) Let $P\left(v_{V}\right)=\left\{(z, t) \in H^{n+1}: z \in A\left(v_{V}\right)\right\}$. The sets $\mathcal{A}(v)=P\left(v_{V}\right) \cap K$ and their images $\mathcal{A}(g v):=g \mathcal{A}(v)$ will be called the $v$-components. The $v$-components form a tessellation of $H^{n+1}$ since the sets $g K, g \in G / G_{\infty}$, do. We shall say that the union of all $\mathcal{A}(g u), g \in G, u \in \bar{D}$, such that $g u=v$ is a $v$-cell $N(v)$. For any $g \in G$, the set $N(g v):=g N(v)$ will be also called a $v$-cell. The tessellation of $H^{n+1}$ by the $v$-cells $N(g v)$ will be called the Farey tessellation of $H^{n+1}$ associated with $G$. Note that the Farey tessellation is $G$-invariant and it is independent of the choice of $P_{\infty}$ (see (1)).

Let $v$ be a vertex of $K$ and let $G_{\infty, v}$ be the stabilizer of $v$ in $G_{\infty}$. Denote $A^{\prime}\left(v_{V}\right)=A\left(v_{V}\right) / G_{\infty, v}$ and $\mathcal{A}^{\prime}(v)=\mathcal{A}(v) / G_{\infty, v}$. The union of non-congruent modulo $G_{\infty}$ polytopes $p\left(u_{i}\right)$ (and $A^{\prime}\left(v_{V}\right)$ ) is a fundamental domain of $G_{\infty}$ in $V$. 


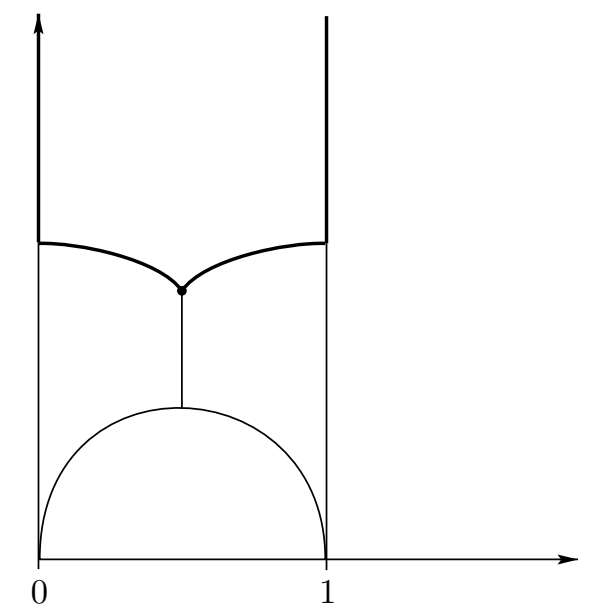

FiguRE 1

Hence if $v_{j}, j=1, \ldots, m$, is a complete set of vertices of $K$ which are not congruent modulo $G_{\infty}$, then the union of the sets $\mathcal{A}^{\prime}\left(v_{j}\right)$ is the closure of a fundamental domain $D$ of $G$ in $H^{n+1}$. By taking if necessary the union of all $\mathcal{A}^{\prime}(v)$, where $v$ runs through the set of all the vertices of the fundamental domain defined by (1) which are not congruent modulo $G_{\infty}$, we can always assume that $D$ is connected.

Assume that $\beta$ is a vertical face of $\mathcal{A}(v)$ which lies in the vertical plane in $H^{n+1}$ through the points $u_{i}=g_{i}(\infty), i=1, \ldots, k$. Then the geodesic $\sigma$ which is the intersection of the isometric spheres of $g_{i}, i=1, \ldots, k$, is orthogonal to $\beta$, and $\beta$ passes through the summit of $\sigma$. It follows that any face $B$ of $N(g v)$ consists of the images of vertical faces of $v$-components. Hence $B$ is the image of some vertical face of $N(v), v \in \bar{D}$. A face $B$ of $N(g v)$ and the projection of $B$ from $\infty$ into $V$ will be called a hyperbolic Farey polytope and Farey polytope respectively. A Farey set of order $m$ is the union of the sets of vertices of $v$-cells $N(g v), g \in G$, such that any vertical line which passes through $D$ passes through at most $m v$-cells between $\infty$ and $N(g v)$.

When the isometric fundamental domain $D$ of $G$ has only one vertex $v \neq \infty$, $\mathcal{A}^{\prime}(v)=\bar{D}$, and the $v$-cell $N(v)$ is a fundamental domain of some subgroup $G_{F}$ of $G$ of index $|\operatorname{Stab}(v, G)|$.

Example 3. Let $n=1$ and $G=S L_{2}(\mathbf{Z})$ (cf. [26]). Then $v$ is an elliptic fixed point of $G$ of order 3, the $v$-component $\mathcal{A}(v)=\bar{D}$, and $G_{F}$ is the subgroup of $G$ of index 3 whose fundamental domain $N(v)$ is the triangle in $H^{2}$ with vertices at $0,1, \infty$ (see Figure 1). The Farey sets are the standard Farey sets for the set of rational numbers (see e.g. [16]).

Example 4. Let $n=1$ and $G$ be the Hecke group $G_{q}$ (cf. [15], [34], [36]). Then $v$ is an elliptic fixed point of $G$ of order $q$, the set $\mathcal{A}(v)=\bar{D}$, and $G_{F}$ is the subgroup of $G$ of index $q$ whose fundamental domain $N(v)$ is the polygon in $H^{2}$ with vertices at $R^{i}(\infty), i=0,1, \ldots, q-1$, where $R \in G_{q}$ is a generator of $\operatorname{Stab}\left(v, G_{q}\right)$ (see Figures 1,2 and 3 where $q=3,4$ and 6 respectively). These groups $G_{F}$ were introduced by A. Haas and C. Series in [15]. 


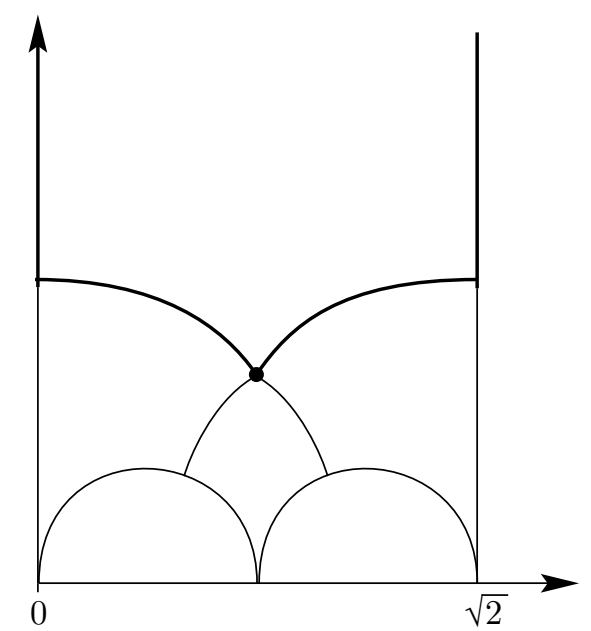

Figure 2

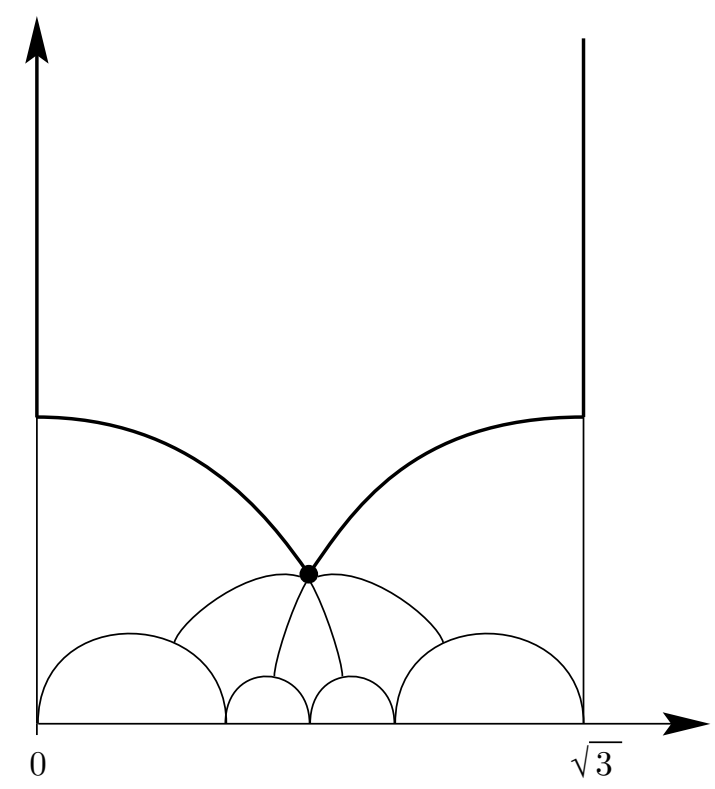

Figure 3

Let $g \in G$. For any $k>0$, let $\mathcal{R}(g, k)$ be the open Euclidean ball in $H^{n+1}$ tangent to $V$ at $g(\infty)$ having radius $r^{2} / k$ where $r=r(g)$ is the radius of the isometric sphere $I(g)$ of $g \in G$. We have $\mathcal{R}(g, k)=g\left(\mathcal{R}_{k}\right)$ where $\mathcal{R}_{k}=\mathcal{R}(i d, k)=\left\{(z, t) \in H^{n+1}\right.$ : $t \geq k / 2\}$. Denote by $Q(g, k)$ and $Q_{k}$ the boundaries of the horoballs $\mathcal{R}(g, k)$ and $\mathcal{R}_{k}$ respectively, and by $\mathcal{N}(k)$ the region in $H^{n+1} \cup \mathcal{P}$ which is exterior to all $\mathcal{R}(g, k)$, $g \in G$.

Let $\alpha \in V-\mathcal{P}$ and $L=(\infty, \alpha)$. Then the inequality (4) holds if and only if $L$ cuts the horosphere $Q(g, k)$. 
A subset $N(g v, k)$ of $N(g v)$ bounded by the horospheres $Q(h, k)$ such that $h(\infty)$ is a vertex of $N(g v)$ is the $k$-neighborhood of $g v$ (cf. [34], [35], [36]). Thus, $N(g v)=$ $N(g v, \infty)$. The region $\mathcal{N}(k)$ is covered by the images of the $k$-neighborhoods $N(v, k), v \in \bar{D}$. There are two kinds of faces of $N(v, k)$ : parts of horospheres $Q(g, k), g \in G$, which will be called the horospherical faces of $N(v, k)$, and the $g e$ odesic faces which are the images of the parts of the vertical faces of $N(v), v \in \bar{D}$.

Let $\sigma$ be the geodesic through $v$ which is perpendicular to a vertical face $B$ of a $v$-cell $N(v)$. Denote $h_{B}=2 h t(\sigma)$. By definition,

$$
k_{G}=\inf h_{B},
$$

the infimum being taken over the set of vertical faces $B$ of all $v$-cells $N(v), v \in \bar{D}$. In the following statement, some of the properties of the sets $N(v)$ and $N(v, k)$ are enumerated. All of them follow from the definitions of these sets.

Theorem 5. 1. Adjacent horospherical and geodesic faces of $N(v, k)$ are orthogonal.

2. The bases of horospherical faces of $N(v, k)$ adjacent to its geodesic face $B$ are the vertices of the face of the $v$-cell $N(v)$ which contains $B$. The converse is also true.

3. Let $\sigma$ be a geodesic through $v$ perpendicular to a vertical face $B$ of $N(v)$. Let $\sigma^{\prime}=g(\sigma), v^{\prime}=g(v)$, and $B^{\prime}=g(B)$ for some $g \in G$. Then all the horospherical faces of $N\left(v^{\prime}, h_{B}\right)$ adjacent to $B^{\prime}$ are tangent to the geodesic $\sigma^{\prime}$ at the point of intersection of $\sigma^{\prime}$ and $B^{\prime}$.

4. Every face of a $v$-cell $N(g v), g \in G$, is the image of a vertical face of some $v$-cell $N\left(v^{\prime}\right), v^{\prime} \in \bar{D}$.

5. Let $B$ be a vertical face of a $v$-cell $N(v)$. Let $k<h_{B}$. If a geodesic $L$ in $H^{n+1}$ cuts a face $B^{\prime}=h(B), h \in G$, then $L$ cuts a horosphere $Q(g, k)$, where $g(\infty)$ is a vertex of $B^{\prime}$.

Proof. 4. If $h(\infty)$ is a vertex of a face $B$ of $N(g v)$, then $h^{-1} B$ is a vertical face of $h^{-1} N(g v)$.

5. Horoballs $\left.\overline{\mathcal{R}}\left(g, h_{B}\right)\right)$ cover $B^{\prime}$.

Remark. It follows from Theorem 5.3 that all the faces of $N\left(g v, k_{G}\right)$ are horospherical which implies Theorem 1 since the vertical line $L=(\infty, \alpha)$ cuts infinitely many $v$-cells $N(g v)$.

Since the boundary of a Farey polytope $F$ is the projection of the boundary of a face of some $v$-cell $N(g v)$, which lies in an $(n-1)$-dimensional hemisphere orthogonal to $V$, the faces of $F$ lie in Euclidean $(n-1)$-planes in $V$. Assume that $\infty$ is not a vertex of $N(g v)$. Since $N(g v)$ is convex and bounded by hemispheres with centers in $V$ or vertical planes, it is bounded from above by one face and all other non-vertical faces of $N(g v)$ form its lower boundary. This leads to the natural subdivision of a Farey polytope into other Farey polytopes (cf. [21], Theorem 4).

Lemma 6. Let Farey polytope $F$ be the projection of the upper boundary of a $v$ cell $N(g v)$. Let $m$ and $m_{v}$ be the numbers of faces and of vertical faces of $N(g v)$ respectively. Then $F$ is subdivided into $m-m_{v}-1$ Farey polytopes, the projections of faces of $N(g v)$ which form its lower boundary.

Example 7. Let $n=2$ and $G$ be the Bianchi group $G L_{2}\left(O_{d}\right)$ where $O_{d}$ is the ring of integers of the imaginary quadratic number field $\mathbf{Q}(\sqrt{-d})$, or extended Bianchi 
group (see [35], [33]). For $d=1,2,3,7$, and 11, there is only one vertex $v$ and $\mathcal{A}^{\prime}(v)=\bar{D}$. If $d=19$, then there are two vertices. For these values of $d$, the spherical groups $G_{v}=\operatorname{Stab}(v, G)$ and the orbits $G_{v} \infty$, which are the sets of the vertices of the $v$-cell $N(v)$, are given in [14]. For $d=1,2,3$, and 7, the projections of $N(v)$ into $V=\mathbf{C}$, which are the Farey polygons, can be found in [21], Figures $1-4$, and for $d=11$, in [24]. Note that in [21] and [24] a different definition of the Farey polygons is given.

Denote by $k_{F}$ the smallest $k$ such that any geodesic of height $k / 2$ in $H^{n+1}$ cuts a vertical face of some $v$-cell $N(h v), v \in \bar{D}, h \in G_{\infty}$.

Lemma 8. If a geodesic $L$ in $H^{n+1}$ cuts a horosphere $Q\left(g, k_{F}\right)$ then $g(\infty)$ is a vertex of a face of some $v$-cell $N(h v), h \in G_{\infty}$, which is cut by $L$.

Proof. Assume that $L$ cuts a horosphere $Q\left(g, k_{F}\right)$. Denote $L^{\prime}=g^{-1}(L)$. Then $h t\left(L^{\prime}\right)>k_{F} / 2$ and, by definition, $L^{\prime}$ cuts a vertical face $B$ of some $v$-cell $N(h v), h \in$ $G_{\infty}$. Thus, $L=g\left(L^{\prime}\right)$ cuts the face $g(B)$ having $g(\infty)$ as one of its vertices.

Let $\alpha \in V-\mathcal{P}$. Let $L=(\infty, \alpha)$. Applying Lemma 8 to the geodesic $L$ we obtain the following statement which contains the basic property of Farey polytopes (cf. [21], Theorem 3).

Theorem 9. Let $\alpha \in V-\mathcal{P}$. If the inequality (4) holds with $k=k_{F}$, then $\alpha$ belongs to a Farey polytope having $g(\infty)$ as one of its vertices.

Let $B$ be a vertical face of a v-cell $N(v)$ and let a Farey polytope $F$ be the projection of $h(B), h \in G$, into $V$. If $\alpha \in F$, then (4) holds with $k=h_{B}$ for some vertex $g(\infty)$ of $F$.

Proof. Let the face $B$ be the same as in the proof of Lemma 8. The Farey polytope which is the projection of $g(B)$ into $V$ contains $\alpha$.

The second statement follows from Theorem 5.5.

Remark. If $\alpha \in V-\mathcal{P}$, then the vertical geodesic $L=(\infty, \alpha)$ in $H^{n+1}$ passes through infinitely many $v$-cells $N(g v)$. Hence there is a sequence of Farey polytopes $F_{i}$ which contain $\alpha$ and such that $F_{i+1} \subset F_{i}, i=1,2, \ldots$, and $\lim _{i \rightarrow \infty} F_{i}=\alpha$. Since, by Theorem 9 , at least one of the vertices of every $F_{i}$ satisfies the inequality (4) with $k=k_{G}=\inf h_{B}$, the infimum being taken over the set of vertical faces $B$ of all $v$-cells $N(v), v \in \bar{D}$, this inequality has infinitely many solutions with $k=k_{G}$. Thus, Theorem 9 implies Theorem 1.

In applications, it is easier to use another definition of $k_{F}$ which is equivalent to the one given above: $k_{F}$ is the largest value of $k$ such that there is a geodesic of height $k / 2$ in $H^{n+1}$ which does not cut a vertical face of any $v$-cell $N(h v), v \in$ $\bar{D}, h \in G_{\infty}$.

Example 10. Let $n=1$ and $G$ be the Hecke group $G_{q}$. In that case, the geodesic with endpoints at 0 and $2 \cos (\pi / q)$ is the highest geodesic which does not cut the vertical faces of $N(v)$ (see Figures 1,2, and 3). Thus, $k_{F}=2 \cos (\pi / q)$.

Example 11. Let $n=2$ and $G$ be a Bianchi group $P G L_{2}\left(O_{d}\right)$. First suppose that the fundamental domain $D$ of $G$ has only one vertex $v \neq \infty$ (see Example 4). If $d=3,7$, or 11 , the projections of the vertical faces of the $v$-cell $N(v)$ form the triangle with vertices at 0,1 , and $\omega$, where $\omega=(1+\sqrt{-d}) / 2$. Figure 1 shows the vertical face of $N(v)$ over the side $[0,1]$ of the triangle. (Note that, for $d=1$, 
the vertical cross section of $N(v)$ over $(0,1+i)$ is shown in Figure 2.) It is easily seen that $k_{F}$ is the diameter of the circumscribed circle of the triangle. Hence, $k_{F}=2 / \sqrt{3}, 4 / \sqrt{7}, 6 / \sqrt{11}$ for $d=3,7,11$ respectively. If $d=1$ or 2 , then the projections of the vertical faces of $N(v)$ form the rectangle with vertices at $0,1, \sqrt{-d}$ and $1+\sqrt{-d}$. Again, $k_{F}$ is the diameter of the circumscribed circle of the rectangle. Thus, $k_{F}=\sqrt{2}, \sqrt{3}$ for $d=1,2$ respectively (cf. [21], Theorem 3). If $d=19$, then $D$ has two vertices $v$ and $v^{\prime}$ (see [14] or [28]). The projections of vertical boundaries of $N(v)$ and $N\left(v^{\prime}\right)$ form the triangle with vertices at $\omega, \omega / 2$, and $(1+\omega) / 2$ and trapezoid with vertices at $0,1, \omega / 2$, and $(1+\omega) / 2$. Thus, $k_{F}$ is the diameter of the circumscribed circle for the trapezoid. Therefore, $k_{F}=\sqrt{35 / 19}$.

Example 12. Let $G$ be the discrete subgroup $\operatorname{SV}\left(\mathbf{Z}^{N}\right), N=2^{n-1}$, of the Vahlen's group of Clifford matrices which is considered in [36] (see also [18]). If $n=1,2,3$, or $4, D$ has only one vertex $v$, the projections of the vertical boundaries of $N(v)$ are the boundary of the unit cube, and $k_{F}$ equals the length of the diagonal of the cube. Thus, $k_{F}=\sqrt{n}$ for $n \leq 4$. For $n=1$ and 2 , these values are found in Examples 10 and 11.

\section{Continued Fractions}

In this section, we introduce Algorithm I which can be used to generate the best possible approximations of elements of the $n$-dimensional euclidean space $V$ by the cusps $g(\infty), g \in G$. This algorithm is similar to the standard continued fraction algorithm and the algorithms used by A. L. Schmidt in [23], [24], and [25]. The properties of Algorithm I are studied and it is applied to develop the reduction theory for geodesics in $H^{n+1}$ which is similar to the reduction theory of binary indefinite quadratic forms.

Let $u=g(\infty)$ be a cusp of the fundamental domain $g(D)$. The set $K(u)$ (see $(2))$ is closely related to the horoballs $\mathcal{R}(g, k)$. Evidently, if a horoball $\mathcal{R}_{k}, t=k / 2$, belongs to $K(\infty)$, then $\mathcal{R}(g, k) \subset K(g \infty)$ for any $g \in G$. And if $\infty$ is the only cusp of $D$, then there is $k^{\prime}>0$ such that $K(g \infty) \subset \mathcal{R}\left(g, k^{\prime}\right)$ for any $g \in G$. It is clear that $\bigcup K(u)=H^{n+1}, u \in G \infty$, and that $\operatorname{dim}\left(K(u) \cap K\left(u^{\prime}\right)\right) \leq n$ if $u \neq u^{\prime}$.

Lemma 13. Assume that a geodesic $L$ passes through the intersection of $K\left(u^{\prime}\right)$, $u^{\prime}=g^{\prime}(\infty)$ and a horoball $\mathcal{R}(g, k)$. Then $L$ cuts $Q\left(g^{\prime}, k\right)$.

Proof. Let $u^{\prime} \neq u=g(\infty)$. Let $z \in K\left(u^{\prime}\right) \cap \mathcal{R}(g, k)$. Suppose that the geodesic interval $M$ with endpoints at $u$ and $z$ passes through $K_{1}, K_{2}, \ldots, K_{m}$, where $K_{1}=K(u)$ and $K_{m}=K\left(u^{\prime}\right)$, in the indicated order. Denote by $S_{1}$ the sphere which contains the common boundary of $K_{1}$ and $K_{2}=K\left(g_{2} \infty\right)$. Then $\mathcal{R}\left(g_{2}, k\right)$ is the image of $\mathcal{R}(g, k)$ under reflection with respect to $S$ and it contains the part of $\mathcal{R}(g, k)$ which is outside the sphere $S$. Hence, $\mathcal{R}\left(g_{2}, k\right)$ contains the part of $M$ with the endpoints at $z$ and $z_{1}=M \cap S$. This argument can be continued to show that $z \in \mathcal{R}\left(g^{\prime}, k\right)$.

Let $\alpha \in V-\mathcal{P}$ and let $L=(\infty, \alpha)$. Suppose that the geodesic $L$ passes through the sets $K(\infty), K\left(u_{1}\right), \ldots, K\left(u_{i}\right), \ldots, u_{i}=g_{i}(\infty), g_{i} \in G$, in the indicated order.

Corollary 14. Let $\alpha \in V-\mathcal{P}$ and let $L$ be the vertical ray through $\alpha$ in $H^{n+1}$. Then

$$
k(\alpha)=2 \lim _{i \rightarrow \infty} \sup h t\left(g_{i}^{-1} L\right)=\lim _{i \rightarrow \infty} \sup \left|g_{i}^{-1}(\infty)-g_{i}^{-1}(\alpha)\right|
$$

where $g_{i} \in G$ are defined as above. 
Denote

$$
\lambda_{i}=L \cap K\left(u_{i}\right), u_{i}=g_{i}(\infty) .
$$

The (continued fraction) Algorithm I can be used to find the sequence $\left\{g_{i}\right\} \subset G$ mentioned above explicitly. The corresponding shift operator is defined on the sequence

$$
\lambda_{1}, \lambda_{2}, \ldots, \lambda_{i}, \ldots
$$

Since for any orbit $G z, z \in H^{n+1}$, a point of the largest height in the orbit belongs to the fundamental domain $D$, we can confine ourself to the geodesics which pass through $D$. Recall that the floor of $D$ consists of the non-vertical faces of $D$.

We now introduce the natural orientation of a geodesic $L^{\prime}=g(L)$ (from $g(\infty)$ to $g(\alpha))$. The partition of $L^{\prime}$ into arks $\lambda_{i}^{\prime}$ is defined by (6). It is clear that this partition is invariant under the action of $G$, that is, $\lambda_{i}^{\prime}=g\left(\lambda_{i}\right)$ for all $i$.

We shall say that a geodesic $L^{\prime}$ is reduced if it passes through $D$ and the initial point of $\lambda^{\prime}=L^{\prime} \cap K(\infty)$ lies in the floor of $D$.

Suppose that the floor of $D$ consists of faces $\phi_{1}, \ldots, \phi_{r}$. Let transformation $R_{j} \in G$ be such that

\section{Algorithm I.}

$$
\phi_{j}=\bar{D} \cap R_{j}^{-1}(\bar{D}), \quad j=1, \ldots, r .
$$

Step 0. Suppose that $\alpha \in U_{0}(P), U_{0} \in G_{\infty}$. Denote $L_{0}^{\prime}=U_{0}^{-1}(L)$. Clearly, $L_{0}^{\prime}$ cuts the floor of $D$ and it is not reduced.

Step 1. Let $t_{1} \in \phi_{j}$ be the point of intersection of $L_{0}^{\prime}$ with the floor of $D$. Denote $S_{0}=R_{j}, g_{0}=T_{0}=U_{0} S_{0}$, and $L_{1}=S_{0}^{-1} L_{0}^{\prime}$. (If $L$ passes through the boundary of two or more faces in the floor of $D, S_{0}$ is not unique.) Then $L=g_{0} L_{1}$ where $L_{1}$ is reduced.

Assume that the elements $T_{1}, \ldots, T_{i-1}$ in $G$ are determined. Let $g_{k}=g_{k-1} T_{k}$ and $L_{k}=T_{k} L_{k+1}, k=1, \ldots, i-1$. Then $L=T_{0} \ldots T_{i-1} L_{i}$.

Step $i+1$. Let $\lambda_{i}=\left(t_{i}, t_{i+1}\right)=L_{i} \cap K(\infty)$. Let $L_{i}^{\prime}=U_{i}^{-1}\left(L_{i}\right)$ where $U_{i} \in G_{\infty}$ is determined so that $U_{i}^{-1} t_{i+1}$ lies in a face $\phi_{k}$ of the floor of $D$. Denote $S_{i}=$ $R_{k}, T_{i}=U_{i} S_{i}$, and $L_{i+1}=S_{i}^{-1} L_{i}^{\prime}$ where a unique $S_{i} \in G$ is such that $S_{i} A_{1}^{\prime} \in \bar{D}$. (If $L_{i}$ passes through the boundary of two or more faces of $D, S_{i}$ is not unique.) Then

and

$$
g_{i}:=g_{i-1} T_{i}
$$

$$
L_{i}=T_{i} L_{i+1}, L=T_{0} \ldots T_{i} L_{i+1} .
$$

It is clear that Algorithm I enumerates $g_{i} \in G$ in the same order as $L$ passes through the sets $K\left(g_{i} \infty\right)$, and that there is a 1-1 correspondence between the arcs $\lambda_{i}$ of $L$ and $T_{i} \in G$ as defined by Algorithm I. The cusps $g_{i}(\infty)$ as defined by Algorithm I will be called the convergents of $\alpha$.

All the geodesics $L_{i}$, as well as $L_{i}^{\prime}$ with orientation changed for the opposite, generated by Algorithm I are reduced. Thus, there are two reduced geodesics in the $G$-orbit of $L$ whose $\operatorname{arc} \lambda_{i}=\left(t_{i}, t_{i+1}\right)$ lies in $K(\infty)$ : one with $t_{i}$ and the second with $t_{i+1}$ in the floor of $D$. (If $\lambda_{i} \in D$, then these two geodesics are different only in orientation.) Since the partition of $L$ is invariant with respect to $G$, any reduced geodesic in the $G$-orbit of $L$ belongs to one of these two sequences generated 
by Algorithm I. When applying Algorithm I, to reduce the number of different transformations $R_{i}$ used, we choose $D$ so that the number of faces in the floor of $D$ is as small as possible.

Example 15. Suppose that there is an isometric fundamental domain $D$ for $G$ such that the floor of $D$ lies in the unit sphere $|z|^{2}+t^{2}=1$. Such a fundamental domain exists, for example, when $n=1$ and $G$ is a Hecke group, or $n=2$ and $G$ is a Bianchi group $P G L_{2}\left(\mathcal{O}_{d}\right), d=1,2,3,7,11$, or $G$ is the discrete subgroup $\operatorname{SV}\left(\mathbf{Z}^{N}\right), N=2^{n-1}, n=1,2,3$, or 4, of the Vahlen group (see Example 12). In all these cases, we choose $P_{\infty}$ to be the Dirichlet polygon for $G_{\infty}$ in $V$ with center at the origin.

In that case we can choose $S_{i}=S$ to be the reflection with respect to this unit sphere. Thus, $S x=(\bar{x})^{-1}$ for $x \in H^{n+1}$. Here ${ }^{-}$is the conjugation in the Clifford algebra so that $x \bar{x}=|x|^{2}$. Since $U_{i}=U\left(a_{i}\right)=\left(\begin{array}{cc}1 & a_{i} \\ 0 & 1\end{array}\right)$ where $a_{i}$ belongs to the lattice $\Lambda \in V$, we have

$$
T_{i}=T\left(a_{i}\right)=\left(\begin{array}{cc}
a_{i} & 1 \\
1 & 0
\end{array}\right) \theta
$$

where $\theta$ is the conjugation in the Clifford algebra mentioned above. Let

$$
g_{i}=T_{0} \ldots T_{i}=\left(\begin{array}{cc}
p_{i} & p_{i-1} \\
q_{i} & q_{i-1}
\end{array}\right) .
$$

By definition, the endpoints of the geodesic $L_{i}$ are $g_{i}^{-1}(\infty)=-q_{i-1} q_{i}^{-1}$ and $\alpha_{i+1}=$ $g_{i}^{-1}(\alpha)$. Since $L_{i}$ is reduced,

$$
\left|q_{i-1}\right|<\left|q_{i}\right| \text { and }\left|\alpha_{i+1}\right|>1
$$

It follows that, for any $i$, the inequality

$$
\left|\alpha-p_{i} q_{i}^{-1}\right|<\frac{1}{k\left|q_{i}\right|^{2}}
$$

holds with $k=2 \sin \frac{\pi}{q}$ if $G=G_{q}$, the Hecke group, and with $k=\sqrt{2}$ when $G$ is the Picard group $P G L_{2}\left(O_{1}\right)$. In particular, if $G$ is the modular group $G_{3}$, (8) holds with $k=\sqrt{3}$ for any $i$.

Suppose that a geodesic $L^{\prime}$ passes through a $v$-cell $N(v)$. Let $u=g(\infty)$ be a vertex of $N(v)$. If the cusp $u$ does not belong to a face of $N(v)$ which is cut by $L^{\prime}$, then, by Theorem 9 , the geodesic $g^{-1}\left(L^{\prime}\right)$ does not cut a vertical face of any $N(h v), h \in G_{\infty}$. Hence $2 h t\left(g^{-1} L^{\prime}\right)<k_{F}<k_{G}$ and geodesic $g^{-1}\left(L^{\prime}\right)$ is not extremal (see $\S 4$ for the definition of an extremal geodesic).

Assume that a geodesic $L_{i}^{\prime}$ enters a $v$-cell $N(v)$ through a vertical face $B$ and exits through face $B^{\prime}$. Let $A_{0}, A_{1}, \ldots, A_{r}\left(A_{0} \in \bar{D}\right)$ be all the $v$-components in $N(v)$ which are passed through by $L_{i}^{\prime}$ in the indicated order. Let convergent $u_{j}=g_{i+j}(\infty)$ be the cusp of $A_{j}, j=0, \ldots, r$. We say that $u_{j}$ is a convergent of type $I$ if it belongs neither to the face $B$ nor to $B^{\prime}$. Otherwise, $u_{j}$ is a convergent of type II. Thus, any convergent of type I does not satisfy the inequality (8)) with $k=k_{F}$. Similarly, we shall say that a geodesic $L_{i}$ which passes through $N(v)$ is of type I if it does not cut a vertical face of $N(v)$. Otherwise, $L_{i}$ is of type II.

Since the union of all the $v$-components in $N(v)$ whose cusps belong to $B$ is a convex set, the set of convergents $u_{0}, \ldots, u_{r}$ can be divided into two subsets: the set of convergents of type I $u_{k}, \ldots, u_{m}, 0<k \leq m<r$ (which can be empty), and 
the set of convergents of type II. Any convergent of type II satisfies the inequality (8) with $k=k_{F}$. Moreover, the convergents $u_{0}$ and $u_{r}$ satisfy (8) with $k=k_{G}$ since $L_{i}^{\prime}$ and $L_{i+r}^{\prime}$ cut the vertical faces of $A_{0}$ and $g_{i+r}^{-1}\left(A_{r}\right)$ respectively. We have the following.

Theorem 16. Let $\alpha \in V-\mathcal{P}$. Then $k(\alpha)$ can be found from (5) where the sequence of $g_{i} \in G$ is generated by Algorithm $I$ and $g_{i}(\infty)$ runs through the subsequence of convergents of type II.

Remark. Assume that $\alpha$ lies in the projection of a vertical face $B$ of a $v$-cell $N(v)$ into $V$. Then any convergent $p_{i} q_{i}^{-1}$ for $\alpha$ is of type II and it satisfies (8) with $k=h_{B} \geq k_{G}$ since an arc of $L_{i}$ lies in a vertical face of a $v$-component in $D$. For example, let $n=2$ and $G$ be a Bianchi group $B_{d}$. If the projection of $B$ into $V=\mathbf{C}$ is the interval $[0,1]$ on the real axis (see Figure 1 ), then $h_{B}=\sqrt{3}$. Thus, if $\alpha$ is a real number, then any convergent of $\alpha$ is real and satisfies the inequality (8) with $k=\sqrt{3}$ (cf. Example 15). If the projection of $B$ into $V$ is the interval $[0, \omega]$, then $h_{B}=\sqrt{3}, \sqrt{2}, 1$, for $d=3,7,11$ respectively (see Figures 2 and 3). Thus, the convergents of $\alpha \in \omega \mathbf{R}$ satisfy (8) with $k=h_{B}$. These approximation constants coincide with the constants $k=2 \sin \frac{\pi}{q}$ obtained for the Hecke groups $G_{q}$ for $q=3,4,6$ in Example 15. It happens because, in these cases, $\operatorname{Stab}\left(P_{B}, G\right)$ is isomorphic to the corresponding Hecke group. Here $P_{B}$ is the vertical plane in $H^{3}$ spanned by $B$.

For a $v$-cell $N(v)$, denote by $m(v)$ the largest number of $v$-components in $N(v)$ passed through by a geodesic, and let $m(G)=\max m(v)$, the maximum being taken over all vertices $v \in \bar{D}$. (If $D$ has a cusp in $V$, then $m(G)=\infty$.) Since $L$ passes through infinitely many $v$-cells, the following result, which is an analog of Vahlen's [29] (and/or Borel's [6]) theorem on regular continued fractions, provides an alternative proof of Theorem 1.

Theorem 17. Let $u_{i}, \ldots, u_{i+m(G)-1}$ be a set of consecutive convergents for $\alpha \in$ $V-\mathcal{P}$ where $m(G)$ is defined as above. Then at least one of them satisfies the inequality (8) with $k=k_{G}$.

Example 18. Let $n=1$ and let $G$ be a Hecke group $G_{q}$. Then $k_{G}=2, m\left(G_{q}\right)=$ $[(q+1) / 2]+1$, and no more than four convergents $u_{0}, u_{1}, u_{r-1}, u_{r}$ out of $r+1$ convergents $u_{0}, \ldots, u_{r}$ mentioned above can be of type II. By Theorem 17, for any $\alpha \in V-\mathcal{P}$ at least one of $[(q+1)]$ consecutive convergents satisfies (8) with $k=2$. When $q=3$ or $4,[(q+1)]=2$. For $q=3$ it is an analog of Vahlen's theorem [29]; for $q=4$, of Borel's theorem [6] (cf. [36], Example 1).

Remark. Let $r_{i}$ be the radius of the isometric sphere of $g_{i} \in G$. Since $\mid g_{i}^{-1}(u)-$ $g_{i}^{-1}\left(u^{\prime}\right) \mid=O\left(r_{i}^{-2}\right)$ for any $u, u^{\prime} \in V$, one can expect that the geodesics $L=(u, \alpha)$ and $L^{\prime}=\left(u^{\prime}, \alpha\right)$ will eventually have the same tails. It is easily seen that if $\alpha$ lies on the hemisphere which contains a common boundary $H$ of $K(\infty)$ and $K\left(S_{i}(\infty)\right)$, $u$ lies inside and $u^{\prime}$ outside of $H$, then $L$ and $L^{\prime}$ pass through different sequences of $v$-components. (But if $S_{i}^{2}=i d$, then $S_{i} L$ and $L^{\prime}$ pass through the same sequences. Hence they have the same tails.)

Now let $\eta, \theta \in V-\mathcal{P}$ and $L=(\eta, \theta)$. The Algorithm I can be defined as above but the sequences of reduced geodesics $L_{i}$ as well as the sequences of $T_{i}$ and $g_{i}$ in $G$ are infinite in both directions as $i \rightarrow \infty$ and as $i \rightarrow-\infty$. Now we have

$$
L_{-k}=T_{-k} \cdots T_{0} \cdots T_{i} L_{i+1}
$$


for any non-negative integers $k$ and $i$. If the orientation is changed for the opposite, then geodesics $L_{i}$ are not reduced any more but the geodesics $L_{i}^{\prime}$, defined by Algorithm I, are.

Example 19. Let $n=1$ and let $G$ be the Hecke group $G_{4}$. Let $L=(-1,1)$. We apply Algorithm I to $L^{\prime}=U(\sqrt{2}) L=(\sqrt{2}-1, \sqrt{2}+1)$. If, as in Example 8, $S_{i}=S$ is the reflection with respect to the unit circle with center at the origin, the sequence of reduced geodesics generated by Algorithm I is periodic, and the period consists of two reduced geodesics $L^{\prime}=T(2 \sqrt{2}) T(-2 \sqrt{2}) L^{\prime}$ and $L^{\prime \prime}=T(-2 \sqrt{2}) L^{\prime}$. If, as in the Rosen algorithm [20], we choose $S_{i}=W=\left(\begin{array}{cc}0 & 1 \\ -1 & 0\end{array}\right)$, then the period consists of only one geodesic $L^{\prime}=T L^{\prime}$ where $T=\left(\begin{array}{cc}-2 \sqrt{2} & -1 \\ 1 & 0\end{array}\right)$. Thus, there are exactly two reduced geodesics in the $G$-orbit of $L$.

Let $n=1$ and $G=G L_{2}(\mathbf{Z})$. Let $L=((1-\sqrt{5}) / 2,(1+\sqrt{5}) / 2)$. Choosing $S$ as in Example 15, we have $L=T(1) L$. There are two reduced geodesics $L$ and $S(L)$. If we choose $S_{i}=W$, then $L=T_{0} T_{1} L$ and $L^{\prime}=T_{1} L$, where $T_{0}=U(1) W, T_{1}=$ $U(-1) W$, are reduced.

For $L=(2-\sqrt{3}, 2+\sqrt{3})$, there are only two reduced geodesics in the $G$-orbit of $L: L=T(4) T(-4) L$ and $L^{\prime}=T(-4) L$.

As above, we divide the set of reduced geodesics into subsets of type I and II. The following statements are analogous to the results obtained above.

Theorem 20. Let $L=(\eta, \theta)$ where $\eta, \theta \in V-\mathcal{P}$. If $L_{i}$ is a reduced geodesic of type II, then $2 h t\left(L_{i}\right) \geq k_{F}$.

Moreover, if $L_{i}$ cuts a vertical face of a v-component in $D$, then $2 h t\left(L_{i}\right) \geq k_{G}$.

Theorem 21. Let $L=(\eta, \theta)$ where $\eta, \theta \in V-\mathcal{P}$. Then

$$
k(L)=2 \sup h t\left(L_{i}\right),
$$

the supremum being taken over all geodesics $L_{i}$ of type II.

Let $L=(\eta, \theta)$ be the axis of a loxodromic element $h \in G$. Let $L^{o}$ be a fundamental domain of the cyclic group generated by $h$ on $L$ chosen so that it consists of whole $\operatorname{arcs} \lambda_{1}, \ldots, \lambda_{p}$. Note that $\lambda_{i+p}=h\left(\lambda_{i}\right)$ and $L_{i+p}=L_{i}$ for all $i$. Thus the sequence $T_{i}$, as generated by Algorithm I, is also periodic, $T_{i+p}=T_{i}$ for all $i$, and $h=T_{1} \cdots T_{p}$. Note that if $\operatorname{Stab}(L, G)=\left\langle S, S^{\prime} \mid S^{2}=S^{\prime 2}=i d\right\rangle$, then, with the proper choice of $\lambda_{1}$, there is an additional symmetry $\lambda_{p-i}=S\left(\lambda_{i}\right), i=1, \ldots, p-1$. We have the following.

Theorem 22. The sequence of arcs (7) of a geodesic $L$ is periodic if and only if $L$ is the axis of a loxodromic element. (If $\lambda_{i+p}=\lambda_{i}$ and $h=T_{1} \cdots T_{p}$, then $h(L)=L$.)

Let $L=(\eta, \theta)$ be the axis of a loxodromic element $h \in G$. Suppose that $L=L_{0}$ is reduced. There are only finitely many reduced geodesics $L_{1}, \ldots, L_{p}=L_{0}$ in the $G$-orbit of $L$ and Algorithm I can be used to find all of them. Also,

$$
k(\eta)=k(\theta)=k(L)=2 \sup h t\left(L_{i}\right), 1 \leq i \leq p,
$$

where $L=T_{0} \cdots T_{i} L_{i+1}$ and the sequence $T_{i}$ is generated by Algorithm $I$.

In particular, if the fundamental domain of $\operatorname{Stab}(L, G)$ on $L$ belongs to $K(\infty)$ (in which case $p=1$ or 2), then $k(L)=2 h t(L)$. 
Remark. Let $\mathbf{B}$ be the ball model of the $(n+1)$-dimensional hyperbolic space. Let $G$ be a geometrically finite discrete subgroup of the group of orientation-preserving isometries of $\mathbf{B}$. For some fixed $w \in \mathbf{B}$, let $D(w)$ denote the Dirichlet polytope with center at $w$ and let $K(w)=G_{w} D(w)$. Upon replacing the region $K(\infty)$ by $K(w)$ in the definition of Algorithm I we can define a similar algorithm for the ball model.

\section{EXtremal GeOdesics}

In the rest of this paper, the group $G$, which is originally defined to be a subgroup of orientation-preserving isometries of $H^{n+1}$, will be sometimes extended by reflections, which, in this paper, are the isometries of order two. The set of all points of $H^{n+1}$ fixed by a reflection $R$ is its axis $a_{R}$ and we usually say that $R$ is the reflection with respect to $a_{R}$. The axis $a_{R}$ of a reflection $R$ is a hyperbolic subspace of $H^{n+1}$. If $\operatorname{codim}\left(a_{R}\right)$ is odd, then $R$ is an orientation-reversing isometry of $H^{n+1}$; otherwise $R$ preserves orientation. When $n=2$, an element $R \in B_{d} \subset G$ such that $\operatorname{tr}(R)=0$ will be simply called a reflection. In that case, the axis $a_{R}$ of $R$ is a geodesic in $H^{3}$ and $R$ reverses the orientation in any hemisphere in $H^{3}$ through $a_{R}$.

A geodesic $L$ in $H^{n+1}$ with the endpoints at $\eta, \eta^{\prime} \in V$ is said to be extremal if

$$
k(L)=2 \sup h t(g L)=\left|\eta-\eta^{\prime}\right|, g \in G .
$$

Algorithm I can be used to find an extremal geodesic in the $G$-orbit of $L$ since the set of reduced geodesics for $L$ contains an extremal geodesic if it exists. By Theorem 22, if $L$ is the axis of a loxodromic element in $G$, then the number of reduced geodesics is finite and an extremal geodesic in the $G$-orbit exists. Let $L$ be extremal. Then $h_{L}=2 h t(L)=k(L) \in \mathcal{M}(G)$. Since the Hurwitz constant $C(G) \geq 1 / k(L)$ for any geodesic $L$, we have obtained a lower bound for $C(G)$. Thus, by Theorem 1 ,

$$
1 / k(L) \leq C(G) \leq 1 / k_{G}
$$

and if a critical edge of $D$ lies on the extremal geodesic $L$, then $k_{G}=k(L)$ and $C(G)=1 / k_{G}$ in Theorem 1 .

Here simple criteria of extremality of a geodesic (see Corollary 24 and Lemma 25) are applied to some Bianchi groups. For each extremal geodesic found, Algorithm I is used to find the set of reduced geodesics. In conclusion, it is shown that if the endpoints $v$ and $v^{\prime}$ of a critical edge $\sigma$ of $D$ are cusps and there is a reflection $R \in G$ such that $v^{\prime}=R(v)$, then the Hurwitz constant $C(G)=k(\sigma)=\left|v^{\prime}-v\right|$ and it is an accumulation point in the Lagrange and Markov spectra (Corollary 30 ).

It is clear that an extremal geodesic $L$ does not cut any horosphere $Q\left(g, h_{L}\right)$. (Otherwise, $h t\left(g^{-1} L\right)>h t(L)$.) As above, let $L^{o}$ be the fundamental domain of $\operatorname{Stab}(L, G)$ on $L$. Assume that $L^{o}$ passes through the sets $K\left(u_{i}\right), u_{i}=g_{i}(\infty), i=$ $1, \ldots, r$. We have the following.

Lemma 23. The following statements are equivalent:

1. A geodesic $L$ is extremal.

2. $L^{o}$ belongs to the closure of $\mathcal{N}\left(h_{L}\right)$.

3. $L^{o}$ cuts none of the horospheres $Q\left(g_{i}, h_{L}\right), 1, \ldots, r$.

In particular, we have the following.

Corollary 24. If $L^{o} \subset K(\infty)$ then $L$ is extremal. 
In the examples below, besides Corollary 24, the following simple criterion of extremality of a geodesic will be used.

Lemma 25. Let $B$ be a vertical face of a v-cell $N(v)$ and let $s \in B$ be the foot of the perpendicular from $v$ into $B$. Assume that there are reflections $R \in G_{s}$ and $W \in G_{v}$ such that the axis $a_{R}$ of $R$ lies in $B$ and $a_{R}$ and the axis of $W$ do not meet.

If the axis $L$ of the loxodromic element $\Phi=R W \in G$ cuts the vertical face of $\mathcal{A}(v)$ which lies in $B$, then $L$ is an extremal geodesic.

Proof. Since the fundamental domain of $\operatorname{Stab}(L, G)$ on $L$ lies in $N(v), L$ is extremal if and only if it cuts none of the horospheres $Q\left(g_{i}, h_{L}\right), g_{i}(\infty)$ being a vertex of $N(v)$. Since $W \in \operatorname{Stab}(L, G)$, we can confine ourself to those horospheres whose bases belong to $B$. (For others, $2 h t\left(g_{i}^{-1} L\right)<k_{F}$.) Assume that $g_{i}(\infty) \in B$. Since $Q=Q\left(g_{i}, k\right)$ and $L$ both are orthogonal to $a_{R}, L$ cuts $Q$ if and only if the point $b=L \cap a_{R}$ is inside $Q$. If $b$ belongs to the vertical face of $A(v)$, then $h_{L} \geq h_{B}$. By the definition of $N\left(v, h_{L}\right)$, since $b \in \mathcal{A}\left(v, h_{L}\right) \subset N\left(v, h_{L}\right)$, none of the horoballs $\mathcal{R}\left(g_{i}, h_{L}\right)$ contains $b$. Thus, $L$ is extremal.

Theorem 5.5 implies the following.

Lemma 26. Suppose that a geodesic $L$ meets a face $g B, g \in G$, where $B$ is a vertical face of $N(v)$. If $h t(L)<h_{B} / 2$, then $L$ is not extremal.

Let $n=2$ and $G$ be a Bianchi group. For the vertical faces $B$ of $v$-cells shown in Figures 1-3 we have $h_{B}=\sqrt{3}, \sqrt{2}, 1$ respectively. By Lemma 26, if geodesic $L$ cuts a triangular face $B$ of a $v$-cell, then $k(L) \leq \sqrt{3}$. Thus, if $k(L)>\sqrt{3}, L$ does not cut a hyperbolic Farey triangle. When $d=2$ or 7 there is up to $G$-equivalence only one extremal geodesic which does not cut the triangles whereas when $d=11$ there are infinitely many such geodesics (see [22], [24], [32]).

Suppose that $\sigma$, a critical edge of $D$ with the endpoints $v, v^{\prime} \in H^{n+1}$ and summit $s$, lies on a geodesic $L$. The groups $G_{v}$ and $G_{v^{\prime}}$ are spherical. If each of them contains reflections which fix $L$, then $\sigma$ contains a fundamental domain of $\operatorname{Stab}(L, G)$ on $L$. Hence, by Corollary $24, L$ is extremal, and the Hurwitz constant $C(G)=1 / k_{G}$. It is known (see [10], p. 226) that the group of symmetries of a regular polytope contains a reflection with respect to its center unless it is a dihedral group of order $2 q, q$ odd, or the tetrahedral group.

On the other hand, for $n=2$, if $G_{v}$ is a dihedral group of order $2 q, q$ odd, and $L$ is an axis of symmetry of order two or the tetrahedral group, then $L$ cuts a horospherical face of $N\left(v, k_{G}\right)$. Hence, $L$ is not extremal and $C(G)<1 / k_{G}$. It explains why, when $G$ is a Bianchi group $P G L_{2}\left(O_{d}\right)$, the equality $C(G)=1 / k_{G}$ holds for $d=1$ or 2 but does not hold when $d \equiv 3(\bmod 4)($ see [14], [7], [35]). The case when $G$ is a Hecke group $G_{q}$ is considered in [34], [36]. In that case, $G_{v}$ is a dihedral group of order $2 q$ and the equality $C(G)=1 / k_{G}$ holds if and only if $q$ is even.

When applying the results obtained to Diophantine approximation in $V$ we can use the well known results on the structure of the discrete spherical groups (see e.g. [4]).

When $n=1$ and $G_{v}$ is a finite or infinite dihedral group, all extremal geodesics $L$ for which $L \cap N(v)$ contains a fundamental domain of $\operatorname{Stab}(L, G)$ on $L$ are enumerated in [34]. In the Examples 27, 28, and 31 below we apply Corollary 24 and 
Lemma 25 to find such extremal geodesics for some Bianchi groups $P G L_{2}\left(O_{d}\right)$ with the generator $x \rightarrow \bar{x}, x \in V=\mathbf{C}$, adjoined.

Denote by $\mathcal{H}_{n}$ the dihedral group, and by $A_{4}$ and $S_{4}$ the tetrahedron and cube symmetry groups respectively. The isometric fundamental domains for these groups for square-free $d \leq 19$ are described in [5], [28]. In [7], for $d=1,2,3,6$ and 7, the vertex and edge subgroups of $G$ are given as well as the presentation of $G$ as a graph amalgamation product. In [14], for $d=1,2,3,7,11$ and 19, the groups $G_{v}$ are described and the orbits $G_{v}(\infty)$ are found. We shall use these results in the examples below. In Examples 27, 28 and $\S 5$, the following notation will be used: $J, J^{\prime \prime}$ are the reflections in the vertical planes in $H^{3}$ through the imaginary and real axes in $V=\mathbf{C}$ respectively; $S$ is the reflection in the unit sphere in $H^{3}$ with center at the origin in $\mathbf{C} ; R(b), b \in O_{d}$, is the reflection in the vertical line through $b / 2 ; W=S J$. We choose $D$ so that $J(D)=D$ and enumerate the extremal geodesics up to this symmetry.

Example 27. When $d=3,7$ or 11, the fundamental domain $D$ has only one vertex $v \neq \infty$. The stabilizer of $v$ is $A_{4}$ for $d=3$ and 11 , and for $d=7, G_{v}=\mathcal{H}_{3}$ (see [5], [28], [14], [7]). We choose $D$ so that $v$ lies above the imaginary axis. We have

$$
\begin{aligned}
& W=\left(\begin{array}{cc}
0 & 1 \\
-1 & 0
\end{array}\right), R(b)=\left(\begin{array}{cc}
-1 & b \\
0 & 1
\end{array}\right), \\
& R^{\prime}=\left(\begin{array}{ll}
-1 & 0 \\
-\bar{\omega} & 1
\end{array}\right), R^{\prime \prime}=\left(\begin{array}{cc}
a & \omega(1-a) \\
\bar{\omega} & -a
\end{array}\right),
\end{aligned}
$$

where $R^{\prime}=S R S, R^{\prime \prime}=R R^{\prime} R, a=|\omega|_{-1}^{2}$, and we abbreviate $R=R(\omega)$. Let $R_{1}=$ $J R J, R_{1}^{\prime}=W R W, R_{1}^{\prime \prime}=R_{1} R_{1}^{\prime} R_{1}$. Then $R, R^{\prime}, R^{\prime \prime} \in G_{s}$ and $R_{1}, R_{1}^{\prime}, R_{1}^{\prime \prime} \in G_{s^{\prime}}$. Here, points $s$ and $s^{\prime}=J s$ in $H^{3}$ are the summits of the critical edges of $D$. They lie above $\omega / 2$ and $-\bar{\omega} / 2$ respectively. Let

$$
\begin{aligned}
\Phi_{1} & =\left(\begin{array}{cc}
0 & 1 \\
1 & -\omega
\end{array}\right), \Phi_{2}=\left(\begin{array}{cc}
\bar{\omega} & -2 \\
-2 & \omega
\end{array}\right), \\
\Phi_{3} & =\left(\begin{array}{cc}
0 & 1 \\
1 & -2 \omega
\end{array}\right), \Phi_{4}=\left(\begin{array}{cc}
1 & -\omega \\
-\omega & \omega^{2}-1
\end{array}\right) .
\end{aligned}
$$

Since the axis of the reflection $W$ does not meet the axes of $R$ and $R_{1}$, the elements $\Phi_{1}=W R$ and $\Phi_{1}^{\prime}=W R^{\prime}=R_{1} W$ are loxodromic, and their axes $L_{1}$ and $L_{1}^{\prime}$ are extremal by Corollary 24. When $d=3$, the axes of $W, R^{\prime \prime}$, and $R_{1}^{\prime \prime}$ meet. For $d=7, R^{\prime}=R^{\prime \prime}$ and $R_{1}^{\prime}=R_{1}^{\prime \prime}$. When $d=11$, the axes of $W$ and $R^{\prime \prime}$ do not meet and the axis $L_{2}$ of $\Phi_{2}=W R^{\prime \prime}$ is extremal by Corollary 24 . The endpoints $\eta, \eta^{\prime} \in V$ of $L_{1}$ are the roots of $f_{1}(x)=x^{2}-\omega x-1=0$ and $k\left(L_{1}\right)=k(\eta)=\left|\eta-\eta^{\prime}\right|=\left|\omega^{2}+4\right|$. Thus, $k\left(L_{1}\right)=k\left(L_{1}^{\prime}\right)=13^{1 / 4}, 8^{1 / 4}$, and $5^{1 / 4}$ for $d=3,7$ and 11 respectively. Similarly, $k\left(L_{2}\right)=\sqrt{5} / 2$. Notice that $1 / k\left(L_{1}\right)$ is the Hurwitz constant for $d=3$ and 7 , and $1 / k\left(L_{2}\right)$ is when $d=11$ (see e.g. [35]).

Denote $u=(\omega, 1) \in H^{3}$. The reflections $R_{2}=R(2 \omega)$ and $R_{2}^{\prime}=R R(0) W R$ belong to $G_{u}$. The axis of $W$ does not meet the axes of $R_{2}$ and $R_{2}^{\prime}$. The axes $L_{3}, L_{4}$ of the loxodromic elements $\Phi_{3}=W R_{2}$ and $\Phi_{4}=W R_{2}^{\prime}$ respectively are extremal by Lemma 25 .

We have $k\left(L_{3}\right)=2\left|\omega^{2}+1\right|^{1 / 2}$ and $k\left(L_{4}\right)=\left|\omega^{4}+4\right|^{1 / 2} /|\omega|$. When $d=3,1 / k\left(L_{4}\right)$ $=13^{-1 / 4}$ and $1 / k\left(L_{3}\right)=1 / 2$ are the first and second minima in the Lagrange 
spectrum. For $d=7,1 / k\left(L_{4}\right)=3^{-1 / 2}$ is the second minimum in the Lagrange spectrum (see e.g. [21]).

In all the cases above, when applying Algorithm I, we can choose $D$ as in Example 15 so that its floor lies in the unit sphere with center at the origin and $S_{i}=W$. The sequence of reduced geodesics obtained is periodic. The length of the period is two and the number of reduced geodesics is two if the reflection $W$ fixes a reduced geodesic and it is equal to four if $W$ does not.

Let $L=L_{1}$. Then there are exactly two reduced geodesics in the $G$-orbit of $L$ : $L=T_{0} T_{1} L$ and $L^{\prime}=T_{1} L$ where $T_{0}=U(\omega) W, T_{1}=U(-\omega) W$.

Let $d=11$ and $L=L_{2}$. Since an arc of $L$ lies in the floor of $D, L$ is not reduced. But $L^{\prime}=R(L)$ is. Let $T_{0}=U(\omega) W, T_{1}=U(\bar{\omega}) W$. There are four reduced geodesics: $L^{\prime}=T_{0} T_{1} L^{\prime}, L^{\prime \prime}=T_{1} L^{\prime}$, and $W\left(L^{\prime}\right), W\left(L^{\prime \prime}\right)$ with their orientation changed for the opposite.

The geodesic $L=L_{3}$ is reduced. Let $T_{0}=U(2 \omega) W, T_{1}=U(-2 \omega) W$. There are exactly two reduced geodesics $L=T_{0} T_{1} L$ and $L^{\prime}=T_{1} L$.

Let $d=7$ and $L=L_{4} . \quad L$ is reduced. Denote $T_{0}=U(\sqrt{-7}) W, T_{1}=$ $U(-\sqrt{-7}) W$. There are exactly two reduced geodesics $L=T_{0} T_{1} L$ and $L^{\prime}=T_{1} L$.

Example 28. Let $G$ be the extended Bianchi group $B_{15}$ or $B_{19}=P G L_{2}\left(O_{19}\right)$ with the generator $x \rightarrow \bar{x}, x \in V=\mathbf{C}$, adjoined (see [33] or [35]). The fundamental domain $D$ is bounded by the unit spheres in $H^{3}$ with centers at $0, \omega,-\bar{\omega} \in V$, by the spheres of radius $r$ with centers at $\omega / 2$ and $-\bar{\omega} / 2$ where $r=1 / \sqrt{2}$ if $d=15$ and $r=1 / 2$ if $d=19$, and by vertical planes through the sides of the triangle with vertices at $0, \omega$, and $-\bar{\omega}$. $D$ is divided into two $v$-components by the vertical plane through the points $\omega / 2$ and $-\bar{\omega} / 2$ in $V$.

First let $d=19$. There are two vertices $v=(4 / \sqrt{-19}, \sqrt{3 / 19}), w=(6 / \sqrt{-19}$, $\sqrt{2 / 19})$ and $\operatorname{Stab}(v, G)=\mathcal{H}_{3}$ and $\operatorname{Stab}(w, G)=A_{4}$ (see e.g. [14]). Let

$W^{\prime}=\left(\begin{array}{cc}2 & -\omega \\ \bar{\omega} & -2\end{array}\right), \Phi_{2}=\left(\begin{array}{cc}-2 & \omega \\ -\bar{\omega} & 3\end{array}\right), \Phi_{3}=\left(\begin{array}{cc}2 & \omega \\ \bar{\omega} & 2\end{array}\right), \Phi_{5}=\left(\begin{array}{cc}-3 & \omega+2 \\ \omega & 2-\omega\end{array}\right)$

$W^{\prime \prime}=R W^{\prime} R, W_{1}^{\prime}=J W^{\prime} J$ and $W_{1}^{\prime \prime}=J W^{\prime \prime} J$. Let the reflections $W, R, R_{1}, R_{2}$ be defined as in Example 27. Then $W, W^{\prime}, W_{1}^{\prime} \in G_{v}$ and $W^{\prime \prime}, W_{1}^{\prime \prime} \in G_{w}$. The axes of reflections $R$ and $R(0)$ pass through the vertical face of $\mathcal{A}(v)$ which is orthogonal to the edge $\sigma$ of $D\left(\sigma\right.$ lies on the axis of $\left.W^{\prime}\right)$. By Corollary 24, the axes $L_{1}, L_{2}, L_{3}$ of the loxodromic elements $\Phi_{1}=W R, \Phi_{2}=W^{\prime} R$ and $\Phi_{3}=W^{\prime} R(0)$ respectively are extremal. (On the other hand, the axis $L_{4}$ of $W_{1}^{\prime} R$ with $h t\left(L_{4}\right)=5^{-1 / 4} / 2$ is not extremal since it does not cut $K(\infty)$.) Thus, we have $k\left(L_{1}\right)=5^{1 / 4}, k\left(L_{2}\right)=1$, and $k\left(L_{3}\right)=2$.

Consider now the $v$-cell $N(w)$. The reflections $W^{\prime \prime}=R W^{\prime} R, W_{1}^{\prime \prime}=J W_{1}^{\prime} J$ belong to $G_{w}$. The axes of $R$ and $R_{2}$ pass through the vertical face of $\mathcal{A}(w)$ which is orthogonal to the edge $\sigma^{\prime}$ of $D\left(\sigma^{\prime}\right.$ lies on the axis of $\left.W^{\prime \prime}\right)$. By Lemma 25, the axes $L_{2}, L_{5}, L_{3}^{\prime}$ of the loxodromic elements $W^{\prime \prime} R=R W^{\prime}, \Phi_{5}=W_{1}^{\prime \prime} R$ and $W^{\prime \prime} R_{2}=R W^{\prime} R(0) R$ respectively are extremal. Thus, we have $k\left(L_{2}\right)=1, k\left(L_{5}\right)=$ $(9 / 5)^{1 / 4}=1.15829$, and $k\left(L_{3}^{\prime}\right)=2$. Note that the Hurwitz constant $C\left(B_{19}\right)=$ $1 / k\left(L_{2}\right)=1$.

Now let $d=15$. The extended Bianchi group $B_{15}$ is not generated by reflections (see $[27])$. The fundamental domain $D$ consists of two $v$-components $\mathcal{A}(v)$ and 
$\mathcal{A}(w)$ where $v=(-3 / \sqrt{-15}, \sqrt{2 / 5})$ and $w=(\sqrt{-15} / 3,1 / \sqrt{3})$. Denote

$$
W^{\prime}=\frac{1}{\sqrt{3}}\left(\begin{array}{cc}
-1-\bar{\omega} & 1+\omega \\
1+\omega & 1+\bar{\omega}
\end{array}\right), \Phi_{7}=\left(\begin{array}{cc}
1 & -1-\omega \\
1+\bar{\omega} & -5
\end{array}\right),
$$

$W_{1}^{\prime}=R W^{\prime}, W^{\prime \prime}=J W^{\prime} J, W_{1}^{\prime \prime}=J W_{1}^{\prime} J, S^{\prime}=R S R$, and $S^{\prime \prime}=R_{1} S R_{1}$. Then $S, W, W^{\prime}, W^{\prime \prime} \in G_{v}=\mathcal{H}_{2}$ and $S^{\prime}, S^{\prime \prime}, W_{1}^{\prime}, W_{1}^{\prime \prime} \in G_{w}=\mathcal{H}_{3}$. Note that $R, W^{\prime}, W_{1}^{\prime} \in$ $\operatorname{Stab}(u, G)$, the Klein 4-group, where $u=(\omega / 2,1 / \sqrt{2})$. The reflection $W^{\prime}$ and loxodromic elements $W R$ and $W^{\prime \prime} R$ have the common axis $L_{1}$ which is an extremal geodesic with $k\left(L_{1}\right)=\sqrt{2}$. The reflection $W$ and loxodromic elements $W^{\prime} R(0)$ and $W^{\prime \prime} R(0)$ also have the common axis $L_{3}$ which is an extremal geodesic with $k\left(L_{3}\right)=2$. Let $J^{\prime}=R J R$ be the reflection in the vertical plane in $H^{3}$ through the line $\operatorname{Re} z=1 / 2$. The reflection $W_{1}^{\prime}$ and loxodromic elements $J^{\prime} \Phi_{7} J^{\prime}, S^{\prime \prime} R$ and $S^{\prime \prime} W^{\prime}$ have the common axis $L_{7}^{\prime}$ which is an extremal geodesic with $k\left(L_{7}\right)=$ $k\left(L_{7}^{\prime}\right)=k\left(J\left(L_{7}^{\prime}\right)\right)=\sqrt{2}$ where $L_{7}$ is the axis of $\Phi_{7}$. In $N(w)$, the axes of $W_{1}^{\prime \prime}$ and $R, W_{1}^{\prime}$ and $R_{2}$, and $W_{1}^{\prime}$ and $R_{2}^{\prime}$ do not meet. Hence the axes $L_{6}, L_{3}$ and $L_{4}$ of $W_{1}^{\prime \prime} R, W_{1}^{\prime} R_{2}$ and $W_{1}^{\prime} R_{2}^{\prime}$ respectively are extremal and $k\left(L_{6}\right)=6^{1 / 4}, k\left(L_{3}\right)=2$, and $k\left(L_{4}\right)=3^{1 / 2}$. We find that $k_{G}=\sqrt{7} / 2$ and, since the critical edges of $D$ are not extremal, $1 / \sqrt{2} \leq C\left(B_{15}\right)<2 / \sqrt{7}$. (It will be shown in $\S 5$ that $C\left(B_{15}\right)=1 / \sqrt{2}$.)

Finally, let $L=L_{8}$ be the geodesic through the vertices $w_{1}=R w$ and $w_{2}=R_{1} w$. The endpoints of $L_{8}$ are the roots of $f_{8}(x)=3 x^{2}-i \sqrt{15} x-3=0$. The stabilizer of $L_{8}$ is generated by $W$ and $W_{2}=J^{\prime} W_{1}^{\prime} J^{\prime}$. Let $s$ be the point of intersection of the axis of $W$ with $L_{8}$. Then the arc of $L_{8}$ with endpoints $s$ and $w_{1}$ is a fundamental domain of $\operatorname{Stab}\left(L_{8}, G\right)$. It cuts the common face of $N(v)$ and $N\left(w_{1}\right)$ with vertices $0, \omega / 2$ and $\infty$. Since $L_{8}$ does not cut the horospheres with bases at these vertices when $k=h_{L}=\sqrt{21} / 3$, by Lemma $23(2), L_{8}$ is extremal and $k\left(L_{8}\right)=\sqrt{21} / 3$.

When applying Algorithm I we choose $D$ so that the floor of $D$ consists of three faces which lie on the spheres with centers at the points $0, \omega / 2$, and $\bar{\omega} / 2$ in $V$, and we define Algorithm I so that $S_{i} \in\left\{W, W^{\prime}, \bar{W}^{\prime}\right\}$ for any $i$. Figure 4 shows the upper one-half of the projection of $D$ from $\infty$ into $\mathbf{C}$.

If $L=L_{1}$, then, as in Example 27, there are two reduced geodesics in the $G$-orbit of $L: L=T_{0} T_{1} L$ and $L^{\prime}=T_{1} L$ where $T_{0}=U(\omega) W, T_{1}=U(-\omega) W$.

Let $d=19$. The geodesic $L=L_{2}$ lies in the floor of $D$ and it is reduced. Let $T_{0}=$ $U(\omega) W, T_{1}=U(\bar{\omega}) W$. There are four reduced geodesics: $L=T_{0} T_{1} L, L^{\prime}=T_{1} L$, and $W(L), W\left(L^{\prime}\right)$ with their orientation changed for the opposite (cf. Example 27, $\left.d=11, L=L_{2}\right)$.

Let $d=15$ and $L=L_{7}=R J\left(L_{7}^{\prime}\right)$. The endpoints of $L_{7}$ lie in $(1+\omega) \mathbf{R}$ and it is the axis of $\Phi_{7}$. Let $T_{0}=U(1+\omega) W, T_{1}=U(1+\bar{\omega}) W$. There are four reduced geodesics: $L=T_{0} T_{1} L, L^{\prime}=T_{1} L$, and $W(L), W\left(L^{\prime}\right)$ with their orientation changed for the opposite.

Let $L=L_{3}$. Then, for $d=19$, there are four reduced geodesics in the $G$-orbit of $L: L=W \bar{W}^{\prime} W W^{\prime}(L), \bar{W}^{\prime} W W^{\prime}(L), W W^{\prime}(L)$, and $W^{\prime}(L)$; and there are only two $L=\bar{W}^{\prime} W^{\prime}(L)$, and $W^{\prime}(L)$ when $d=15$.

Let $\sigma$ be an edge of $D$ whose endpoints $v$ and $v^{\prime}$ are cusps of $D$. For $n=1$, it was shown in [34] that the geodesic $L \supset \sigma$ is extremal and $k(L)$ is not isolated in the Lagrange spectrum provided there is a reflection $R \in G_{s}$ such that $v^{\prime}=R v$. (Here $s$ is the summit of $\sigma$.) We shall generalize this result to higher dimensional spaces. 


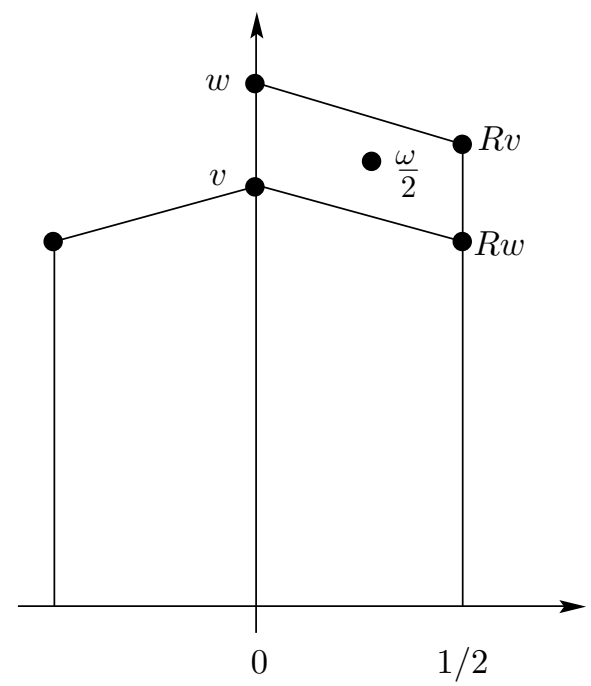

Figure 4

Theorem 29. Suppose that $v$ is a cusp of D. Let $B$ be a vertical face of a v-cell $N(v)$ and let $s \in B$ be the foot of the perpendicular from $v$ into $B$. Assume that there are reflections $R \in G_{s}$ and $W \in G_{v}$ such that the axis $a_{R}$ of $R$ lies in $B$ and $a_{R}$ and the axis of $W$ do not meet. Then the axis of the loxodromic element $R W$ as well as the geodesic $L=(v, R(v))$ is extremal. Moreover, $k(L)$ is an accumulation point in the Lagrange and Markov spectra.

Proof. Let $P_{n}$ run through the set of all parabolic elements in $G_{v}$. The axes of the reflections $W_{n}=P_{n}^{-1} W P_{n} \in G_{v}$ converge to $v$ and the axes $L_{n}$ of the loxodromic elements $W_{n} R$ converge to $L$. The sequence of geodesics $L_{n}$ contains a subsequence of geodesics which cut the vertical face of $\mathcal{A}(v)$ that lies in $B$ and, by Lemma 25, they are extremal.

Corollary 30. Assume that the geodesic $L$ in Theorem 29 is a critical edge of $D$. Then the Hurwitz constant $C(G)=1 / k(L)$ and it is an accumulation point in the Lagrange and Markov spectra.

Example 31. Let $G=G P L_{2}\left(O_{15}\right)$. The fundamental domain $D$ of $G$ has the cusp $v=(\omega / 2,0)$ and vertex at $w=(2 \sqrt{-15} / 7, \sqrt{3} / 7) \in H^{3}$. It consists of two $v$-components $\mathcal{A}(v)$ and $\mathcal{A}(w)$, where $\mathcal{A}(v)$ is bounded by vertical planes through the sides of the rhombus with vertices $(0,0), c=(-4 / \sqrt{-15}, 0),(\omega, 0), c^{\prime}=$ $((-7+\sqrt{-15}) / 2 \sqrt{-15}, 0)$, unit spheres with centers at $(0,0$ and $(\omega, 0)$, and spheres with radius $1 / \sqrt{15}$ and centers at $c$ and $c^{\prime}$; and the component $\mathcal{A}(w)$ is bounded by vertical planes through the sides of the triangle with vertices $(\omega, 0),(-\bar{\omega}, 0), c$, unit spheres with centers at $(\omega, 0)$ and $(\bar{\omega}, 0)$ and the sphere with center at $c$ and radius $1 / \sqrt{15}$ (see [5], [28]). Since $c$ is the center of the circumscribed circle of the triangle with vertices $0, \omega,-\bar{\omega}$, there are three critical edges $\sigma, \sigma^{\prime}$, and $\sigma^{\prime \prime}$ with the endpoints at $v$ and $v^{\prime}=(-\bar{\omega} / 2,0), v$ and $w$, and $v^{\prime}$ and $w$ respectively in the fundamental domain $D^{\prime}$ bounded by the vertical planes through the sides of this triangle. Thus we have $k_{G}=1 / 2$. It can be easily shown that the edges 
$\sigma^{\prime}$ and $\sigma^{\prime \prime}$ are not extremal. Let $B$ be the face of $\mathcal{A}(v)$ whose projection into $V$ is the interval with endpoints at $(0,0)$ and $c$. The reflections $W$ and $R$ defined as in Examples 27, 28, and 31 with their roles interchanged satisfy the hypothesis of Theorem 29. Hence the edge $\sigma$ is extremal. By Corollary 30, the Hurwitz constant $C(G)=1 / k_{G}=2$ and it is an accumulation point in $\mathcal{L}(G)$ and $\mathcal{M}(G)$.

Example 32. Let $n=4$ and $G$ be the same as in Example 12. It is shown in [36] that the endpoints of a critical edge $\sigma$ are cusps and that $k_{G}=1$. It can be shown that the conditions of Theorem 29 and Corollary 30 are satisfied and, therefore, $C(G)=1 / k_{G}=1$ and it is a limit point in $\mathcal{L}(G)$ and $\mathcal{M}(G)$.

\section{Diophantine approximation in $\mathbf{Q} \sqrt{-15}$}

In this section, Theorem 2 will be proved. Here $G$ is an extended Bianchi group $B_{15}$. Assume that any two $v$-cells $N\left(v_{i}\right)$ and $N\left(v_{i+1}\right)$ in the sequence $N\left(v_{i}\right), i=$ $1, \ldots, n$, have at least one common edge. Generalizing the definition of $k(v)$ introduced in [34], define $k\left(v_{1}, \ldots, v_{n}\right)$ to be the largest $k$ such that any geodesic passing through $N\left(v_{i}, k\right), i=1, \ldots, n$, cuts a horospherical face of at least one of $N\left(v_{i}, k\right), i=1, \ldots, n$. Assume that a geodesic $L$ passes through $v$-cells $N\left(v_{i}\right), i=1, \ldots, n$. We shall say that $L$ is feasible for this sequence of $v$-cells if $L$ does not cut horospherical faces of $N\left(v_{i}, h_{L}\right), i=1, \ldots, n$. Thus, by definition, $k_{G}<h_{L}=2 h t(L)$ for a feasible $L$.

When $d=15$, there are only two types of $v$-cells: tetrahedra and octahedra (see Example 28). Tetrahedra are congruent to $N(v)$ with vertices $0, \omega / 2,-\bar{\omega} / 2, \infty$ and octahedra to $N(w)$ with vertices $\omega,-\bar{\omega}, \omega / 2,-\bar{\omega} / 2,(-1+2 \omega) / 3, \infty$. Each tetrahedron has common faces with octahedra only. Each octahedron has two common faces with octahedra and six with tetrahedra. Octahedra with common faces have a common axis which is the axis of an elliptic element of order three (see Figure 5).

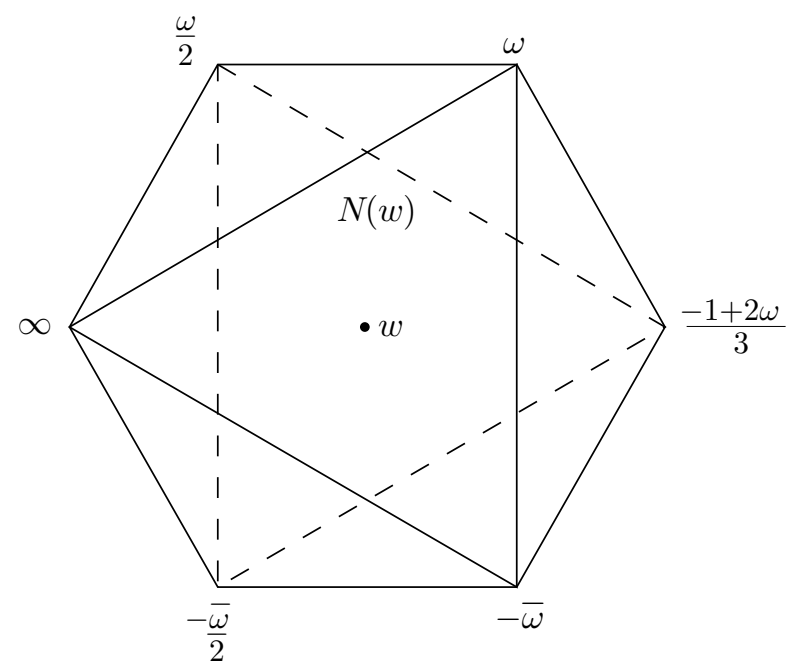

FiguRE 5 
If an extremal geodesic $L$ cuts such a common face (which is congruent to the triangle with vertices $0,1, \infty)$, then $k(L) \geq \sqrt{3}$. Thus,

$$
k\left(w_{1}, J^{\prime \prime} w_{1}\right)=\sqrt{3} .
$$

If a geodesic $L$ meets the axis $a_{R}$ of the reflection $R$ and it does not cut the horospheres with bases at $\omega / 2$ and $\infty$ (which are tangent when $k=\sqrt{2}$ ), then $h_{L} \geq \sqrt{2}$. Since the extremal geodesic $L_{1}$ and $L_{7}^{\prime}$ meet $a_{R}$ and $k\left(L_{1}\right)=k\left(L_{7}^{\prime}\right)=\sqrt{2}$, we have

$$
k(v, R v)=k\left(w, w_{1}\right)=\sqrt{2}
$$

where $w_{1}=R w$. Similarly, if $L$ meets the vertical line through the origin, which is the axis of the reflection $R(0)$, then $h_{L} \geq 2$. Since the axis of $W$ is such a geodesic and it is extremal (see Example 28),

$$
k(v, R(0) v)=k\left(w, R(0) w_{1}\right)=2 .
$$

Assume that $k(L)<\sqrt{2}$. It follows from (9), (10) and (11) that $L$ cannot pass through two tetrahedra or two octahedra in succession. Assume that $L$ passes successively through a tetrahedron $T_{1}$, octahedron $O_{1}$, tetrahedron $T_{2}$, and octahedron $O_{2}$. Let $B$ be the common face of $T_{1}$ and $O_{1}$ and $B^{\prime}$ the common face of $O_{1}$ and $T_{2}$. Let $\tau$ be the reflection in $H^{3}$ with respect to the center of $O_{1}$. It follows that the tetrahedra $T_{1}$ and $T_{2}$ can have (a) a common edge; (b) only one common vertex; or (c) they do not intersect, in which case $\tau(B)=B^{\prime}$. Replacing, if necessary, $L$ by $g(L)$ for some $g \in G$, we can assume that $L$ passes through one of the following sequences of $v$-cells: (a) $N(v), N(w), N(R v)$, or $N(v), N\left(w_{1}\right), N(R v)$; (b) $N(v), N\left(w_{1}\right), N\left(J^{\prime} v\right)$; or (c) $N(v), N\left(w_{1}\right), N(\tau(v))$; and that it is feasible for the corresponding sequence. Here $\tau$ is the reflection with respect to $w_{1}$.

On the other hand, octahedra $O_{1}$ and $O_{2}$ always have a common edge $M$. By applying some $g \in G$, if necessary, we can assume that $M$ is a vertical edge of $N\left(w_{1}\right)$, and that $L$ passes either through $N\left(w_{1}\right), N(v), N(w)$ (when $M$ passes through $\omega / 2$ ) or through $N\left(w_{1}\right), N(v), N\left(J w_{1}\right)$ (when $M$ passes through 0 ).

Lemma 33. $k(v, w, R v) \geq \sqrt{2}, k\left(v, w_{1}, R v\right) \geq \sqrt{2}$.

Proof. Assume that $L$ passes through $N(v)$ and $N(R v)$ and that it is feasible for these $v$-cells. Let $f(x, y)=(x-\theta y)\left(x-\theta^{\prime} y\right)$ where $\theta$ and $\theta^{\prime}$ are the endpoints of $L$. We say that $f$ is extremal if $|f(x, y)| \geq n(x, y)$ for all $x / y=g(\infty), g \in G$. Here $n(x, y)$ is the norm of the ideal generated by $x$ and $y$. It is clear that $f$ is extremal if and only if $L$ is. Let $\Delta=\Delta(f)=\left(\theta-\theta^{\prime}\right)^{2} / 4$. Then $h_{L}=2|\Delta|^{1 / 2}<\sqrt{2}$. Since $k_{G}=\sqrt{7} / 2$ (see Example 28), we can assume that $7 / 16<|\Delta|<1 / 2$. It is clear from geometry (see Figure 6) that $0 \leq \arg (\Delta) \leq 2 \arg (\omega)=4 \alpha$.

Thus, we can assume that

$$
\Delta \in E_{1}:=\{z \in \mathbf{C}: 7 / 16<|z|<1 / 2 ; 0 \leq \arg (z) \leq 2 \arg (\omega)\} .
$$

To show that there are no feasible geodesics with $\Delta \in E_{1}$ we shall utilize the approach which was used in [30] and [31] to find the discrete part of the Markov spectrum for the Gaussian field. Let $b=\left(\theta+\theta^{\prime}\right) / 2$. We can assume that

$$
b \in P:=\{z \in \mathbf{C}: 0 \leq \operatorname{Re}(z) \leq 1 / 2,0 \leq \operatorname{Im}(z) \leq \sqrt{15} / 4\} .
$$

It is clear that $f$ is extremal if and only if $|f(z, 1)|=\left|(z-b)^{2}-\Delta\right| \geq n(x, y)|y|^{-2}$ for any $z=x / y=g(\infty), g \in G$. Thus, if for a fixed $b$ the open disks $K\left(z_{i}\right)$ with 


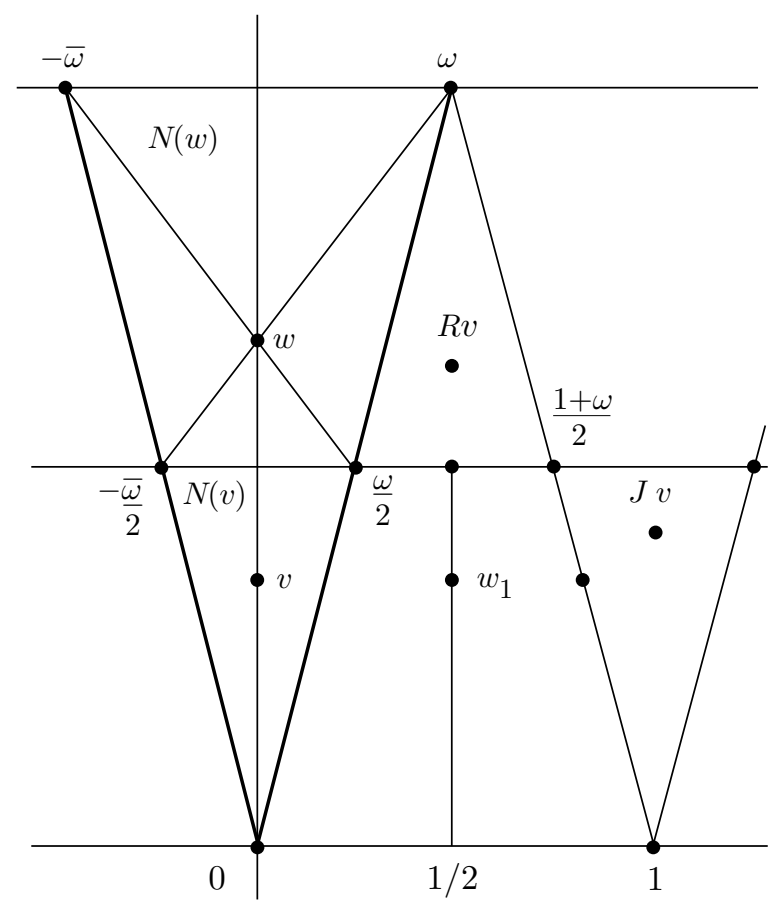

Figure 6

centers at $C\left(z_{i}\right)=\left(z_{i}-b\right)^{2}$ and radii $r\left(z_{i}\right)=n\left(x_{i}, y_{i}\right)\left|y_{i}\right|^{-2}, i=1, \ldots, j$, cover the region $E_{1}$, there are no feasible geodesics with center at $b \in \mathbf{C}$ and $h_{L}<\sqrt{2}$ which cut $N(v)$ and $N(R v)$. We shall show that such a covering exists for any $b \in P$. Only the disks $K(z)$ of radii $r(z)=1$ and $1 / 2$ will be used.

Denote $B_{m}=\left\{z \in \mathbf{C}:\left|z^{2}-1 / 2\right|<1 / m\right\}, m=1,2$. Thus, $B_{1}$ is a convex region bounding a Cassinian oval, and $B_{2}$ is the interior of a lemniscate (these two curves have the same foci $\pm 1 / \sqrt{2}$ ). A part of $B_{2}$ bounded by one leaf of the lemniscate is also convex (see Figure 7).

Let $B_{m}(z, \eta)=z+e^{i \eta} B_{m}$. It is clear that if $b \in B_{m}(z, \eta)$, then $e^{2 i \eta} / 2 \in K(z)$, and, since $r(z) \geq 1 / 2$, if $b \in B_{m}(z, \eta) \cap B_{m}\left(z, \eta^{\prime}\right), 0<\eta<\eta^{\prime}<\pi / 2$, then the arc of the circle $|z|=1 / 2, \eta<\arg (z)<\eta^{\prime}$, is covered by $K(z)$.

Let $a_{1}=1 / 2+i 0.6890, a_{2}=0.4024+i 0.6900, a_{3}=i 0.7070, a_{4}=1 / 2+$ $i 0.7450, a_{5}=i 0.7450, a_{6}=i 0.945$. Divide the rectangle $P$ in (13) into five polygons (see Figure 8): $P_{1}$ is the pentagon with vertices $0,1 / 2, a_{1}, a_{2}, a_{3} ; P_{2}$ is the hexagon with vertices $\omega / 2,1 / 4+\omega / 2, a_{4}, a_{2}, a_{3}, a_{5} ; P_{3}$ is the triangle with vertices $a_{1}, a_{2}, a_{4}, P_{4}$ is the triangle with vertices $a_{5}, a_{6}, \omega / 2$; and $P_{5}$ is the triangle with vertices $a_{6}, i \sqrt{15} / 4, \omega / 2$.

1. Since $P_{1} \subset B_{1}(0, \eta)$ for any $\eta \in[0, \pi / 2]$, and $C(0)=b^{2} / 4, E_{1} \subset K(0)$ if $b \in P_{1}$.

2. Let $b \in P_{2} \subset B_{2}((1+\omega) / 2, \eta) \cap B_{1}\left(0, \eta^{\prime}\right), 0 \leq \eta<\alpha<\eta^{\prime} \leq \pi$. Then $1 / 2, \omega / 4 \in \bar{K}((1+\omega) / 2)$ and $\omega / 4,-1 / 2 \in \bar{K}(0)$. Hence $E_{1} \subset K(0) \cup K((1+\omega) / 2)$.

3. Let $\delta_{1}=e^{i 0.2618} / 2$. Let $b \in P_{3} \subset B_{2}((2+\omega) / 2, \eta) \cap B_{2}\left((1+\omega) / 2, \eta^{\prime}\right) \cap$ $B_{1}\left(0, \eta^{\prime \prime}\right), 0 \leq \eta \leq 0.1309 \leq \eta^{\prime} \leq \alpha \leq \eta^{\prime \prime} \leq 2 \alpha$. Then $|C((1+\omega) / 2)|<1 / 2$ and 


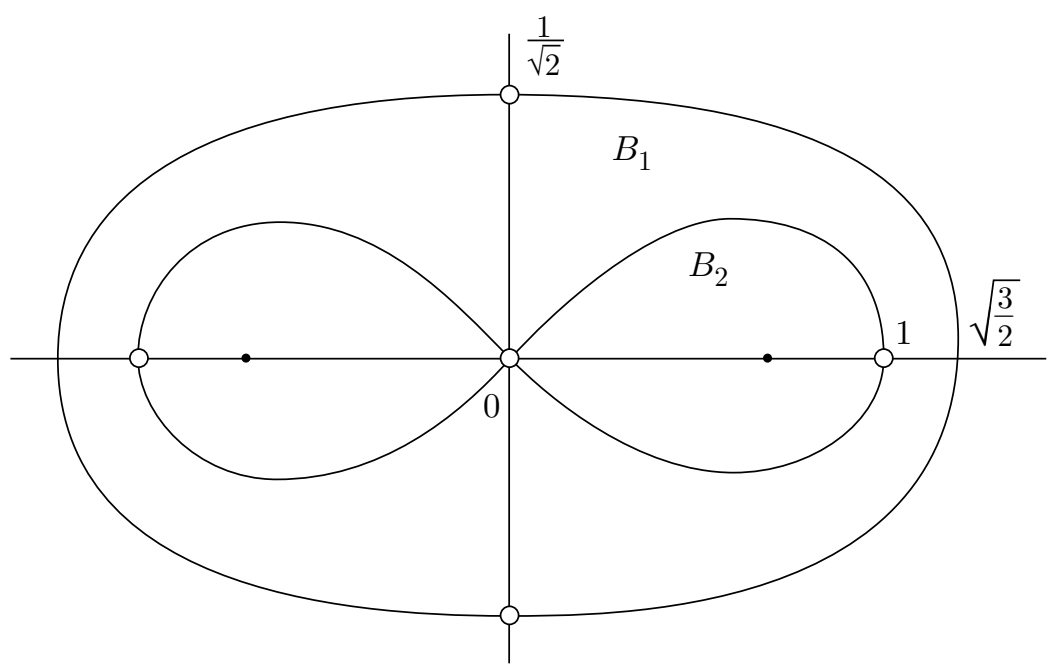

FIGURE 7

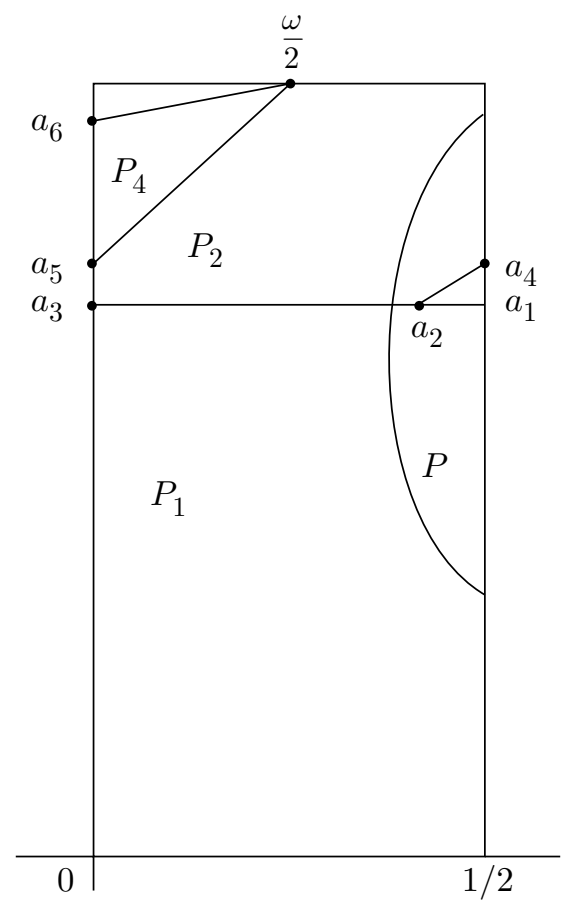

Figure 8

$\omega^{2} / 8, \omega / 4 \in K(0), \omega / 4, \delta_{1} \in K((1+\omega) / 2)$ and $\delta_{1}, 1 / 2,7 / 16 \in K((2+\omega) / 2)$. Thus, $E_{1} \subset K(0) \cup K((1+\omega) / 2) \cup K((2+\omega) / 2)$.

4. Let $\delta_{2}=e^{i 1.658} / 2$. Let $b \in P_{4} \subset B_{2}(\omega / 2, \eta) \cap B_{1}\left(0, \eta^{\prime}\right), 0 \leq \eta \leq 0.827 \leq$ $\eta^{\prime} \leq \pi$. Then $1 / 2, \delta_{2} \in K(\omega / 2) ; \delta_{2},-1 / 2 \in K(0)$, and since $|C(\omega / 2)|<1 / 2, E_{1} \subset$ $K(0) \cup K(\omega / 2)$. 
5. Let $b \in P_{5} \subset B_{2}(\omega / 2, \eta) \cap B_{1}\left(\omega, \eta^{\prime}\right) \cap B_{1}\left(0, \eta^{\prime \prime}\right), 0 \leq \eta \leq 0.827 \leq \eta^{\prime} \leq \pi / 2-\alpha \leq$ $\eta^{\prime \prime} \leq \pi$. Then $1 / 2, \delta_{2} \in K(\omega / 2), i / 2, e^{3 \pi / 4} / 2 \in K(\omega)$, and $-\bar{\omega} / 4,-1 / 2 \in K(0)$. Hence $E_{1} \subset K(0) \cup K(\omega) \cup K(\omega / 2)$.

Thus, for any $b \in P$

$$
E_{1} \subset K(0) \cup K(\omega) \cup K(\omega / 2) \cup K((1+\omega) / 2) \cup K((2+\omega) / 2)
$$

which completes the proof.

Lemma 34. $k\left(v, w_{1}, J^{\prime} v\right) \geq \sqrt{2}$.

Proof. Let $L$ be a feasible geodesic with $h_{L}<\sqrt{2}$ which passes through $N(v)$ and $N\left(J^{\prime} v\right)$. Then $L$ cuts the geodesic faces of $N(v, \sqrt{2})$ and $N\left(J^{\prime} v, \sqrt{2}\right)$ that lie in the vertical faces of $N(v)$ and $N\left(J^{\prime} v\right)$ with vertices $0, \omega / 2, \infty$ and $1,(1+\omega) / 2, \infty$ respectively (see Figures 6 and 9 ). It follows that

$$
\Delta \in E_{2}:=\{z \in \mathbf{C}: 7 / 16<|z|<1 / 2 ;|\arg (z)|<\pi / 3\}
$$

and

$$
b \in P^{\prime}:=\{z \in P:|z-(2+\omega) / 3|<0.45\}
$$

(see Figure 8). By Lemma 33, $E_{2} \cap E_{1}$ is covered by the disks $K(z)$ for any $b \in P^{\prime} \subset P$. Hence it is enough to show that such a covering of $E_{2}^{\prime}=\{z \in$ $\left.E_{2}:-\pi / 3<\arg (z) \leq 0\right\}$ exists for any $b \in P^{\prime}$. Let $P_{6}=P^{\prime} \cap B_{1}(1,0)$ and $P_{7}=P^{\prime}-P_{6}$. If $b \in P_{6} \subset B_{1}(1, \eta),-\pi / 6<\eta \leq 0$, then $E_{2}^{\prime} \subset K(1)$. If $b \in P_{7} \subset B_{2}(-\bar{\omega} / 2, \eta),-\pi / 6<\eta \leq 0$, then $E_{2}^{\prime} \subset K(-\bar{\omega} / 2)$. The lemma is proved.

Remark. Note that $k\left(v, w_{1}\right) \leq k\left(L_{8}\right)=\sqrt{21} / 3$ (see Example 28).

Proof of Theorem 2. It follows from (9), (10), (11), Lemmas 33 and 34 that the inequality $k(L)<\sqrt{2}$ may hold only if $L$ passes through an alternating sequence of polyhedra $\ldots, T_{1}, O_{1}, T_{2}, \ldots$, such that $\tau\left(T_{1}\right)=T_{2}$ for any two consecutive tetrahedra $T_{1}$ and $T_{2}$ in the sequence. Here $\tau$ is the reflection in $H^{3}$ with respect to the center of the octahedron $O_{1}$. As mentioned above, we have to consider two cases.

1. Assume that $L$ passes through $N(w), N(v), N\left(w_{1}\right)$. Then $L$ cuts also $N\left(\tau^{\prime}(v)\right)$ and $N(\tau(v))$ where $\tau^{\prime}$ and $\tau$ are the reflections in $H^{3}$ with respect to $w$ and $w_{1}$ respectively. The projections of $N\left(\tau^{\prime}(v)\right)$ and $N(\tau(v))$ from $\infty$ into $\mathbf{C}$ are the triangles with vertices $\omega,-\bar{\omega},(-1+\omega) / 3$ and $1,(1+\omega) / 2,(1+\omega) / 3$ respectively. But if $L$ passes through $N\left(\tau^{\prime}(v)\right)$ and $N(\tau(v))$, it does not meet $N(v)$ (see Figure 6). The contradiction obtained shows that no such geodesic $L$ exists.

2. Now assume that $L$ passes through $N\left(w_{1}\right), N(v), N\left(J w_{1}\right)$. Then $L$ cuts also $N(\tau(v))$ and $N\left(\tau^{\prime \prime}(v)\right)$ where $\tau^{\prime \prime}=J \tau J$ is the reflection in $H^{3}$ with respect to $J w_{1}$. Let $B$ be the common geodesic face of $N\left(w_{1}, \sqrt{2}\right)$ and $N(\tau(v), \sqrt{2})$ and let $B^{\prime}=J(B)$. ( $B$ is congruent to the geodesic face shown in Figure 9.) Then $L$ cuts both $B$ and $B^{\prime}$. It is clear from geometry that the geodesic $L^{\prime}$ through the vertices $u=((2+\omega) / 4,1 / \sqrt{8})$ and $u^{\prime}=J u=((2-\bar{\omega}) / 4,1 / \sqrt{8})$ of $B$ and $B^{\prime}$ respectively has the smallest height among all the geodesics which cut both $B$ and $B^{\prime}$. But $h t\left(L^{\prime}\right)=\sqrt{33} / 8>1 / \sqrt{2}>h t(L)$ which contradicts the assumption.

Thus, no geodesic with $k(L)<\sqrt{2}$ exists and the Hurwitz constant $C\left(B_{15}\right)=$ $1 / \sqrt{2}$. It is attained at the extremal geodesics $L_{1}$ and $L_{7}^{\prime}$ (see Example 28) whose endpoints are $(\omega \pm \sqrt{\omega}) / 2$ and $(\omega \pm \sqrt{-\omega}) / 2$ respectively. 


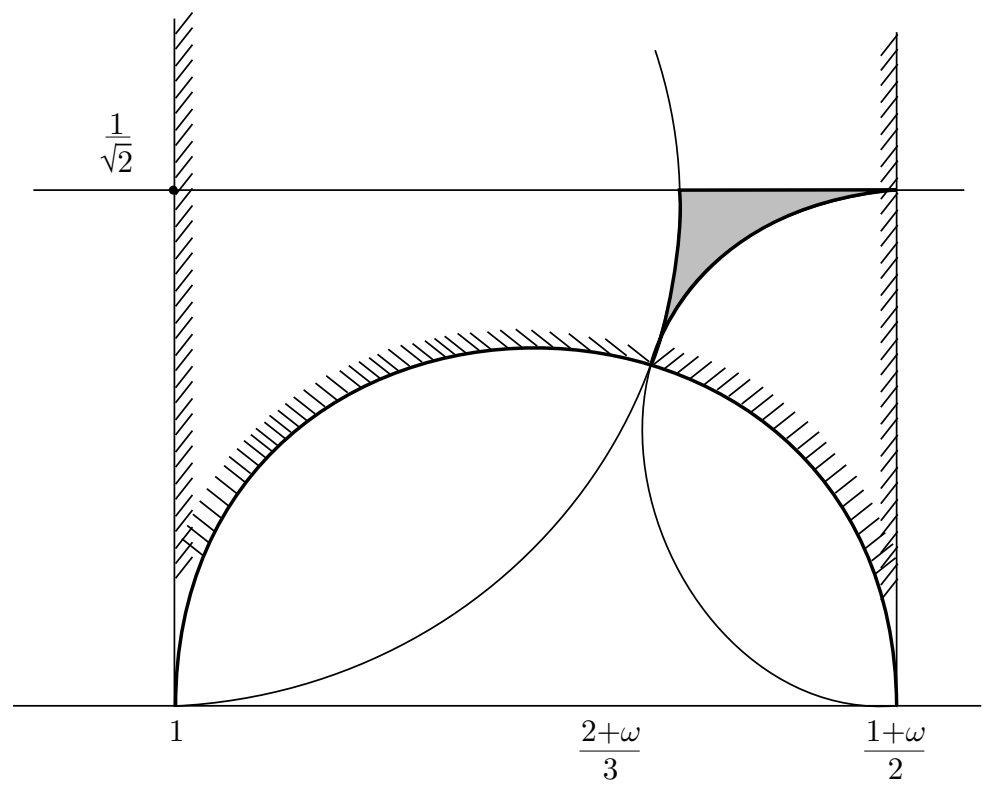

FiguRE 9

\section{ISOLATION THEOREM}

In this section, we generalize to $n$-dimensional euclidean spaces, $n \geq 3$, the isolation theorems proved in [8], p. 25, and [31] for $n=1$ and 2 .

Let $L$ be a geodesic in $H^{n+1}$ with endpoints $\eta$ and $\theta$ in $V$. Let $g \in G$ and let $r=r(g)$ be the radius of the isometric sphere $I(g)$ with center at $x=g^{-1}(\infty) \in V$. Denote

$$
l(g, \eta)=|x-\eta| / r .
$$

Then (see [1], p. 129)

$$
l(g, \eta)=\left|g^{\prime}(\eta)\right|^{-1 / 2}
$$

where $g^{\prime}(x)$ is the Jacobian of $g$. Denote

$$
f_{L}(g)=l(g, \eta) l(g, \theta) .
$$

Then (see [19], p. 8)

which implies the following.

$$
f_{L}(g)=\frac{|\eta-\theta|}{|g(\eta)-g(\theta)|}
$$

Lemma 35. Let $L$ be a geodesic in $H^{n+1}$. Then $L$ cuts a horosphere $Q=Q\left(g, h_{L}\right)$ if and only if $f_{L}\left(g^{-1}\right)<1$, and $L$ is tangent to $Q$ if and only if $f_{L}\left(g^{-1}\right)=1$. Thus $L$ is extremal if and only if $f_{L}(g) \geq 1$ for any $g \in G$.

Proof. A geodesic $L$ cuts the horosphere $Q$ if and only if $h t\left(g^{-1}(L)\right)>h_{L} / 2$ which is equivalent to $f_{L}\left(g^{-1}\right)<1$.

Remark. If $G$ is a Vahlen group and $g(\infty)=a c^{-1}, g \in G$, then we also have the following representation: $f_{L}(g)=|\eta c-a||\theta c-a|$. 
Denote by $C(x, \alpha)$ the spherical cone in $V$ with vertex at $\eta$, axis through $x$, and vertex angle $\alpha$. Let $x_{1}, \ldots, x_{n+1}$ be the vertices of a regular tetrahedron in $V$ with center at $\eta$. Since $\eta$ divides a height of the tetrahedron in ratio $n: 1$, the cones $C\left(x_{1}, \alpha\right), \ldots, C\left(x_{n+1}, \alpha\right)$ cover $V$ provided $\alpha \geq \alpha_{n}=\pi / 4+(\arcsin (1 / n)) / 2$.

Suppose now that $L$ is the axis of a loxodromic element $h \in G$. Then $h(\eta)=\eta$ and $h(\theta)=\theta$. Suppose, as we may, that $h^{k}(x) \rightarrow \eta$ as $k \rightarrow \infty$ for any $x \in V, x \neq \theta$. By the chain rule, $(g h)^{\prime}(\eta)=g^{\prime}(\eta) h^{\prime}(\eta)$. Denote $\lambda^{-2}=\left|h^{\prime}(\eta)\right|$. Since $f_{L}\left(g h^{n}\right)=$ $f_{L}(g)$, we have

$$
l\left(g h^{n}, \eta\right)=\lambda^{-n} l(g, \eta), l\left(g h^{n}, \theta\right)=\lambda^{n} l(g, \theta) .
$$

Suppose that the geodesic $L$ is extremal. For every $c=g(\infty) \in V, g \in G$, let $u(c)$ be the unit tangent vector at $\eta$ to the circle through $c, \eta$, and $\theta$ in the direction of $c$. Let points $c_{i}=g_{i}(\infty) \in V, g_{i} \in G, i=1, \ldots, r$, be such that $f_{L}\left(g_{i}^{-1}\right)=1$ and every cone $C(x, \alpha), \alpha>\alpha_{n}$, contains at least one of the vectors $u\left(c_{i}\right), i=1, \ldots, r$. Since the angle between $u\left(h^{k} c_{i}\right)$ and $u\left(h^{k} c_{j}\right)$ does not depend on $k$, every cone $C(x, \alpha), \alpha>\alpha_{n}$, contains an infinite sequence of points $h^{k} c_{i_{k}}, 1 \leq i_{k} \leq r$, for all integer $k$ greater than some fixed positive constant.

Let $H$ be a hemisphere in $H^{n+1}$ orthogonal to $L$ and let $S=H \cap V$. The region $R$ in $V$ bounded by spheres $S$ and $h S$ is a fundamental domain in $V$ of the infinite cyclic subgroup of $\operatorname{Stab}(L, G)$ generated by $h$. Assume that the points $c_{1}, \ldots, c_{r} \in K=R \cup h R \cup \ldots \cup h^{s} R$.

Denote $K_{m}=h^{s m} K$. Let $L^{\prime}$ be a geodesic with endpoints at $\eta^{\prime}$ and $\theta^{\prime}$ such that $\eta^{\prime} \in K_{m}$ for some $m$ and $\left|\eta^{\prime}-\eta\right|<\epsilon,\left|\theta^{\prime}-\theta\right|<\epsilon$ for some $\epsilon>0$. There is $g \in G$ such that $c=g(\infty) \in C\left(\eta^{\prime}, \alpha_{n}\right) \cap K_{m-1}$ and $f_{L}\left(g^{-1}\right)=1$. Then $h^{2 s} c \in K_{m+1}$. Since the distance between $\eta$ and the center of a sphere $S \in V$ with radius $\rho$ is less than $\rho^{2} / h_{L}$, there is a constant $C$ such that

$$
\lambda^{2 s}\left(1-C \epsilon^{2}\right)|\eta-c|<\left|\eta^{\prime}-\eta\right|<\left(1+C \epsilon^{2}\right)|\eta-c|, c=g(\infty),
$$

where fixed $\epsilon>\left|\eta^{\prime}-\eta\right|$. Suppose that $n \geq 2$. Then $\alpha_{n} \leq \pi / 3$. It follows that there is a constant $C^{\prime}$ such that

$$
\left|\eta^{\prime}-c\right|<\left(1-\lambda^{2 s}+\lambda^{4 s}\right)^{1 / 2}|\eta-c|\left(1+C^{\prime} \epsilon^{2}\right) .
$$

Let $\mu<\left(1-\lambda^{2 s}+\lambda^{4 s}\right)^{1 / 2}$. There is a constant $C^{\prime \prime}$ such that

$$
\left|\theta^{\prime}-c\right| \leq|\theta-c|+\left|\theta^{\prime}-\theta\right|<\left(1+C^{\prime \prime} \epsilon\right)|\theta-c| .
$$

Thus, for a sufficiently small $\epsilon, f_{L^{\prime}}\left(g^{-1}\right)=r^{2}\left|\eta^{\prime}-c\right|\left|\theta^{\prime}-c\right|<\mu$.

We have proved the following isolation theorem.

Theorem 36. Suppose that an extremal geodesic $L$ in $H^{n+1}$ with endpoints $\eta$ and $\theta$ in $V$ is the axis of a loxodromic element $h$ in $G$. Let $u(c)$ be the unit tangent vector at $\eta$ to the circle through $c, \eta$, and $\theta$ in the direction of $c$. Suppose that there are points $c_{i}=g_{i}(\infty) \in V, g_{i} \in G$ such that $f_{L}\left(g_{i}^{-1}\right)=1, i=1, \ldots, r$, and every cone $C(x, \alpha), \alpha>\pi / 4+(\arcsin (1 / n)) / 2$, contains at least one of the vectors $u\left(c_{i}\right), i=1, \ldots, r$. Then there are $k^{\prime}>k(L)$ and an $\epsilon>0$ depending only on $L$ such that $k\left(L^{\prime}\right)>k^{\prime}$ for any geodesic $L^{\prime}$ with endpoints at $\eta^{\prime}$ and $\theta^{\prime}$ for which

$$
\left|\eta^{\prime}-\eta\right|<\epsilon,\left|\theta^{\prime}-\theta\right|<\epsilon
$$

Suppose that an extremal geodesic $L$ is the axis of a loxodromic $h \in G$ and that $L$ contains a critical edge $\sigma$ of $D$ which is orthogonal to the vertical face $B$ of $v$-cell $N(v)$. Then the Hurwitz constant $C(G)=1 / k(L)$. Assume that the group 
of exterior automorphisms of $L$ in $G$ acts transitively on the set of vertices of $B$. (We say $g \in G$ is an exterior automorphism of $L$ if $g$ fixes $L$ pointwise.) Then the hypothesis of Theorem 36 is satisfied since, as it is easily seen, for any vertices $c_{i}$ and $c_{j}$ of $B$, the angle between the geodesic $M_{i}$ through $s=\sigma \cap B$ and $c_{i}$ and $M_{j}$ through $s$ and $c_{j}$ is equal to the angle between the circular arcs through $\eta, c_{i}, \theta$ and $\eta, c_{j}, \theta$. Thus, we have the following.

Corollary 37. Suppose that each of the critical edges $\sigma$ of the fundamental domain $D$ of the discrete group $G$ acting in $H^{n+1}$ lies on the axis $L$ of an loxodromic element in $G$ which is an extremal geodesic. Let $\sigma$ be orthogonal to $B$, a vertical face of a $v$-cell. If the group of exterior automorphisms of $L$ acts transitively on the set of the vertices of $B$, then the Hurwitz constant of $G$ is isolated in its Lagrange and Markov spectra.

Proof. By assumption, the Hurwitz constant $C(G)=1 / k(L)$. Since $L$ satisfies Theorem 36, we can confine ourselves to consideration of only extremal geodesics. Assume that for any $\delta>0$ there is an extremal geodesic $L^{\prime}$ such that $k(L)<k\left(L^{\prime}\right)<$ $k(L)+\delta$. Then, as follows from Theorem 5 , for sufficiently small $\delta, L^{\prime}$ cuts the geodesic faces $B_{o} \subset B$ and $B_{o}^{\prime} \subset B^{\prime}$ of $N\left(v, k\left(L^{\prime}\right)\right)$ where $B$ and $B^{\prime}$ are faces of the $v$-cell $N(v)$ cut by some $L$ containing a critical edge of $D$. Thus, the inequalities (14) are satisfied and by Theorem $36 L^{\prime}$ is not extremal which contradicts the assumption. (Note that the diameter of a geodesic face of $N\left(v, k\left(L^{\prime}\right)\right)$ is small and approaches zero as $\delta \rightarrow 0$.)

In all the examples considered in [35] and [36], the group $G$ is generated by reflections. Hence, Corollary 37 is applicable to $G$, and the Hurwitz constants are isolated in the Lagrange and Markov spectra of $G$ when $n=1$ and $G=G_{q}$, the Hecke group with even $q>2$; or $n=2$ and $G=B_{d}$, the extended Bianchi group, $d=1,2,5$, or 6 ; or $n=3$ and $G$ is the discrete $\operatorname{subgroup~} \operatorname{SV}\left(\mathbf{Z}^{4}\right)$, of the Vahlen's group of Clifford matrices (see Example 12); or $n=4$ and $G=$ $\operatorname{PSL}(2, \mathcal{H})$ where $\mathcal{H}$ is the ring of Hurwitz integral quaternions. Some of these or even stronger results are obtained in [15], [23], [21], [30], [31], and [22]. But the isolation of the approximation constants for the imaginary quadratic fields $\mathbf{Q}(\sqrt{-5})$ and $\mathbf{Q}(\sqrt{-6})$, and for the three-dimensional euclidean space is a new result. This isolation phenomenon should be compared with the case when the endpoints of the critical edge $\sigma$ of $D$ are cusps when the Hurwitz constant is a limit point in the spectra $\mathcal{L}(G)$ and $\mathcal{M}(G)$ (see Example 32 ).

\section{REFERENCES}

1. B.N. Apanasov, Discrete Groups in Space and Uniformization Problems, Kluwer Academic Publishers, Dordrecht/Boston/London 1991. MR 93h:57026

2. A.F. Beardon, The Geometry of Discrete Groups, Springer-Verlag, New York, 1983. MR 85d:22026

3. A.F. Beardon and B. Maskit, Limit points of Kleinian groups and finite sided fundamental polyhedra, Acta Math. 132 (1974), 1-12. MR 48:11489

4. C.T.Benson and L.C. Grove, Finite Reflection Groups, Springer-Verlag, 2nd. ed., 1985. MR 85m:20007

5. L. Bianchi, Sui gruppi de sostituzioni lineari con coefficienti appartenenti a corpi quadratici immaginari, Math. Ann. 40 (1892), 332-412.

6. É. Borel, Contribution à l'analyse arithmétique du continu, J. Math Pures Appl. (5) 9 (1903), 329-375. 
7. A.M. Brunner, Y.W. Lee, N.J. Wielenberg, Polyhedral groups and graph amalgamation products, Topology and its Applications, 20 (1985), 289-304. MR 87a:57049

8. J.W.S. Cassels, An introduction to Diophantine approximation, Cambridge Univ. Press, 1957. MR 19:396h

9. J.H Conway, N.J.A. Sloane, Sphere Packings, Lattices and Groups, Springer-Verlag, New York/ Berlin/ Heidelberg/ London/ Paris/ Tokyo, 1988. MR 89a:11067

10. H.S.M. Coxeter, Regular Polytopes, Dover, 1973. MR 51:6554

11. T.W. Cusick and M.E. Flahive, Markoff and Lagrange spectra, Math. Surveys and Monos., vol. 30, Amer. Math. Soc., Providence, R.I., 1989. MR 90i:11069

12. L.R. Ford, A geometric proof of a theorem of Hurwitz, Proc. Edinburgh Math. Soc., 35 (1917), $59-65$.

13. L.R. Ford, On the closeness of approach of complex rational fraction to a complex irrational number, Trans. Amer. Math. Soc., 27 (1925), 146-154.

14. F. Grunewald, A.C. Gushoff, und J. Mennicke, Komplex-quadratische Zahlk'orper kleiner discriminante und pflasterungen des dreidimensionalen hyperbolischen raumes, Geometriae Dedicata 12 (1982), 227-237. MR 83h:51033

15. A. Haas and C. Series, The Hurwitz constant and Diophantine approximation on Hecke groups, J. London Math. Soc. (2) 34 (1986), 219-234. MR 87m:11060

16. G.H. Hardy and E.M. Wright, An introduction to the theory of numbers, Oxford, 1960. MR 16:673c

17. A. Hurwitz, Über die angenaherte Darstellungen der Irrationalzahlen durch rationale Brüche, Math. Ann. 39 (1891), 279-284.

18. C. Maclachlan, P.L. Waterman, and N.J. Weilenberg, Higher dimensional analogues of the modular and Picard groups, Trans. Amer. Math. Soc. 312 (1989), 739-753. MR 90b:11039

19. P.J. Nicholls, The Ergodic Theory of Discrete Groups, London Math. Soc. Lecture Note Ser. 143, Cambridge University Press, 1989. MR 91i:58104

20. D. Rosen, A class of continued fractions associated with certain properly discontinuous groups, Duke Math. J. 21 (1954), 549-562. MR 16:458d

21. Asmus L. Schmidt, Farey triangles and Farey quadrangles in the complex plane, Math. Scand. 21 (1967), 241-295. MR 39:6831

22. Asmus L. Schmidt, Farey simplices in the space of quaternions, Math. Scand. 24 (1969), 31-65. MR 40:7206

23. Asmus L. Schmidt, Diophantine approximation of complex numbers, Acta Math. 134 (1975), 1-85. MR 54:10160

24. Asmus L. Schmidt, Diophantine approximation in the field $\mathbf{Q}\left(i\left(11^{1 / 2}\right)\right)$, J. Number Theory 10 (1978), 151-176. MR 58:5537

25. Asmus L. Schmidt, Diophantine approximation in the Eisensteinian field, J. Number Theory 16 (1983), 169-204. MR 84m:10024

26. C. Series, The modular surface and continued fractions, J. London Math. Soc. (2) 31 (1985), 69-80. MR 87c:58094

27. M.K. Shaiheev, Reflection subgroups in Bianchi groups, Selecta Mathematica Sovietica 9 (1990), 315-322. MR 91j:20118

28. R.G. Swan, Generators and relations for certain special linear groups, Adv. in Math. 6 (1971), 1-77. MR 44:1741

29. K. Th. Vahlen, Über Näherungswerte und Kettenbrüche, J. Reine Angew. Math. 115 (1895), 221-233.

30. L.Ya. Vulakh, The Markov spectrum of imaginary quadratic field $\mathbf{Q}(i \sqrt{D})$, where $D \not \equiv 3$ $(\bmod 4)$ (Russian), Vestnik Moskov. Univ. Ser. 1 Math. Meh. 26 (1971), no. 6, 32-41. MR 45: 1847

31. L.Ya. Vulakh, The Markov spectrum of the Gaussian field (Russian), Izv. Vysš. Učebn. Zaved. Matematika, 129 (1973), no. 2, 26-40. MR 47:6616

32. L.Ya. Vulakh, The accumulation point of the Markov spectrum of the field $\mathbf{Q}(i \sqrt{D})$ (Russian), Vestnik Moskov. Univ. Ser. 1 Math. Meh. 30 (1975), no. 1, 9-11; 31 (1976), no. 5, 125. MR 51:12732; MR 55:5550

33. L.Ya. Vulakh, Maximal Fuchsian Subgroups of Extended Bianchi Groups, in Number Theory with an Emphasis on the Markoff Spectrum (Provo, UT, 1991), 297-310, Lecture Notes in Pure and Appl. Math., 147, Dekker, New York, 1993. MR 94g:11028 
34. L.Ya. Vulakh, On Hurwitz constants for Fuchsian groups, Canad. J. Math. 49 (1997), 405416. MR 98a: 11087

35. L.Ya. Vulakh, Diophantine approximation on Bianchi groups, J. Number Theory, 54 (1995), 73-80. MR 96g: 11076

36. L.Ya. Vulakh, Diophantine approximation in $\mathbf{R}^{n}$, Trans. Amer. Math. Soc. 347 (1995), 573585. MR 95e: 11076

Department of Mathematics, The Cooper Union, 51 Astor Place, New York, New YORK 10003

E-mail address: vulakh@cooper.edu 